ARTICLE

https://doi.org/10.1038/s41467-018-08115-8

\title{
Tyrosyl phosphorylation of KRAS stalls GTPase cycle via alteration of switch I and II conformation
}

\author{
Yoshihito Kano ${ }^{1,2}$, Teklab Gebregiworgis (10 ${ }^{3}$, Christopher B. Marshall ${ }^{3}$, Nikolina Radulovich ${ }^{4}$, Betty P.K. Poon? \\ Jonathan St-Germain ${ }^{3}$, Jonathan D. Cook ${ }^{1}$, Ivette Valencia-Sama ${ }^{1,5}$, Benjamin M.M. Grant ${ }^{3}$, \\ Silvia Gabriela Herrera ${ }^{6}$, Jinmin Miao ${ }^{7}$, Brian Raught ${ }^{3}$, Meredith S. Irwin ${ }^{5}$, Jeffrey E. Lee ${ }^{1}$, Jen Jen Yeh ${ }^{6,8,9}$, \\ Zhong-Yin Zhang7, Ming-Sound Tsao (1) 4, Mitsuhiko Ikura ${ }^{3}$ \& Michael Ohh (1) ${ }^{1,2}$
}

Deregulation of the RAS GTPase cycle due to mutations in the three RAS genes is commonly associated with cancer development. Protein tyrosine phosphatase SHP2 promotes RAF-toMAPK signaling pathway and is an essential factor in RAS-driven oncogenesis. Despite the emergence of SHP2 inhibitors for the treatment of cancers harbouring mutant KRAS, the mechanism underlying SHP2 activation of KRAS signaling remains unclear. Here we report tyrosyl-phosphorylation of endogenous RAS and demonstrate that KRAS phosphorylation via Src on Tyr32 and Tyr64 alters the conformation of switch I and II regions, which stalls multiple steps of the GTPase cycle and impairs binding to effectors. In contrast, SHP2 dephosphorylates KRAS, a process that is required to maintain dynamic canonical KRAS GTPase cycle. Notably, Src- and SHP2-mediated regulation of KRAS activity extends to oncogenic KRAS and the inhibition of SHP2 disrupts the phosphorylation cycle, shifting the equilibrium of the GTPase cycle towards the stalled 'dark state'.

\footnotetext{
${ }^{1}$ Department of Laboratory Medicine and Pathobiology, University of Toronto, 661 University Avenue, Toronto, ON M5G 1M1, Canada. ${ }^{2}$ Department of Biochemistry, University of Toronto, 661 University Avenue, Toronto, ON M5G 1M1, Canada. ${ }^{3}$ Princess Margaret Cancer Centre, University Health Network and Department of Medical Biophysics, University of Toronto, 101 College Street, Toronto, ON M5G 1L7, Canada. ${ }^{4}$ Princess Margaret Cancer Centre, University Health Network and Department of Pathology, University of Toronto, Toronto, ON M5G 1L7, Canada. ${ }^{5}$ Peter Gilgan Centre for Research and Learning, The Hospital for Sick Children, 686 Bay Street, Toronto, ON 5G OA4, Canada. ${ }^{6}$ Lineberger Comprehensive Cancer Center, University of North Carolina, Chapel Hill, NC 27599, USA. ${ }^{7}$ Department of Medicinal Chemistry and Molecular Pharmacology, Center for Cancer Research and Institute for Drug Discovery, Purdue University, 720 Clinic Drive, West Lafayette, IN 47907, USA. ${ }^{8}$ Department of Surgery, University of North Carolina, Chapel Hill, NC 27599, USA. ${ }^{9}$ Department of Pharmacology, University of North Carolina, Chapel Hill, NC 27599, USA. These authors contributed equally: Yoshihito Kano, Teklab Gebregiworgis, Christopher B. Marshall. Correspondence and requests for materials should be addressed to M.O. (email: michael.ohh@utoronto.ca)
} 
$\mathrm{D}$ eregulation of the RAS GTPase cycle due to mutations in $R A S$ is commonly associated with cancer initiation and progression and several developmental syndromes, referred to as "RASopathies"1. While there are three human RAS genes (HRAS, NRAS, and KRAS) that encode highly related 188-189 amino acid proteins, KRAS is the most frequently mutated oncogene in human cancers, accounting for up to $25 \%$ of lung, $40 \%$ of colorectal, and $95 \%$ of pancreatic cancers ${ }^{2}$. RAS is a small GTPase protein that cycles between GDP-loaded inactive and GTP-loaded activated forms, which adopt distinct conformations at switch I (residues 30-38) and switch II (59-72) regions near the nucleotide-binding site ${ }^{3}$. RAS is activated by guanine nucleotide-exchange factors (GEFs) and the GTP-bound form binds and activates effector proteins, such as RAF. RAS activation is terminated by hydrolysis of GTP, which is accelerated by GTPase-activating proteins (GAPs); however, this step of the GTPase cycle is impaired by most oncogenic RAS mutations ${ }^{4}$.

We previously showed that c-Src (henceforth referred to as $\mathrm{Src}$ ) binds to and phosphorylates H/NRAS, which was associated with RAF displacement and the attenuation of downstream mitogenactivated extracellular signal-regulated kinase (MEK)-to-extracellular signal-regulated kinase (ERK) and phosphoinositide-3 kinase-to-AKT signaling 5 . Conversely tyrosyl-phosphorylated H/ NRAS (pH/NRAS) can be dephosphorylated by SHP2 protein tyrosine phosphatase (PTP), which restores H/NRAS binding to RAF and reactivates downstream signaling ${ }^{6}$. We further showed that pharmacologic inhibition of SHP2 activity attenuates the progression of spontaneous glioblastoma in a mutant HRAS knock-in glioma mouse model ${ }^{6}$. These results taken together support the notion that one of the SHP2 functions is as a direct activator of RAS. Subsequently, a recent series of reports have demonstrated that inhibition of SHP2 suppresses the growth of mutant KRAS-driven lung cancer ${ }^{7}$ and pancreatic ductal adenocarcinoma (PDAC) $)^{8,9}$, as well as gastroesophageal cancer with amplification of otherwise wild-type (WT) KRAS ${ }^{10}$. Another proposed model is that inhibition of SHP2 disrupts GTP loading of RAS by the GEF Son of Sevenless 1 (SOS1), suggesting that SHP2 functions by coordinating adaptor proteins on the cell membrane ${ }^{11}$. However, it remains unclear how SHP2 precisely regulates KRAS-to-mitogen-activated protein kinase (MAPK) pathway since oncogenic KRAS mutations confer resistance to GAPs and most would therefore exhibit elevated GTP loading even in the absence of SOS activity ${ }^{4,12}$.

Here, using real-time nuclear magnetic resonance (NMR) and mass spectrometry (MS), we show definitively that KRAS is phosphorylated via Src on Tyr32 and Tyr64, which alters the conformation of switch I and II regions, respectively, negatively impacting every step of the GTPase cycle. We show specifically that tyrosyl phosphorylation of KRAS markedly attenuates its sensitivity to the activities of GEF and GAP and profoundly impairs its binding affinity to the effector RAF. Intrinsic nucleotide exchange was however enhanced, thus GTP-loaded phosphorylated KRAS (pKRAS) accumulated in a dark, "readyto-serve," state that can be rapidly unleashed via dephosphorylation by SHP2 PTP. Notably, common oncogenic KRAS mutants such as G12V and G12D were not recalcitrant to phosphorylation-mediated regulation, and pharmacologic inhibition of SHP2 led to the accumulation of silenced pKRAS, supporting the potential clinical utility of manipulating the Srcand SHP2-mediated phosphorylation cycle of KRAS in the management of KRAS-driven cancers.

\section{Results}

Pharmacologic inhibition of SHP2 reduces PDAC tumor growth. KRAS mutations are detected in $>90 \%$ of PDAC, one of the deadliest cancers without cure or effective treatment ${ }^{13}$, thus a highly relevant cancer type to evaluate the therapeutic utility of SHP2 inhibitors. Here we investigated the effectiveness of a nextgeneration, cell-permeable catalytic inhibitor of SHP2, 11a-14. Notably, a panel of PDAC cell lines, including those harboring the most frequent mutations G12V or G12D as well as PDAC patient-derived xenograft (PDX) cells were sensitive to 11a-1 treatment (Supplementary Fig. 1a, b), which attenuated the level of epidermal growth factor (EGF)-induced pSHP2, pERK, and pAKT (Supplementary Fig. 1c) while increasing the level of cleaved PARP and caspase 9 in a dose-dependent manner (Supplementary Fig. 1d). In addition, molecular inhibition of SHP2 via CRISPR/Cas9-mediated knockout of SHP2 in the PDAC cell line CFPAC1 harboring the KRAS ${ }^{G 12 V}$ mutation markedly attenuated its rate of cell proliferation (Supplementary Fig. 1e). Moreover, PDAC cell lines grown in three-dimensional (3D) culture or as organoids exhibited dose-dependent sensitivity to 11a-1 as well as an allosteric SHP2 inhibitor SHP099 ${ }^{15}$ (Supplementary Fig. 1f, g, h).

We next interrogated the antitumor efficacy of SHP099 in vivo. We first determined the maximum tolerable dose of SHP099 in SCID mice as only nude mice were used in a previous report ${ }^{15}$. We observed no discernable weight loss in $100 \mathrm{mg} / \mathrm{kg}$ treatment group while $150 \mathrm{mg} / \mathrm{kg}$ treatment showed slight weight loss and 200 and $250 \mathrm{mg} / \mathrm{kg}$ treatments were intolerable (Supplementary Fig. 2a, b). SCID mice bearing subcutaneous PDAC xenografts derived from PDX cells OCIP.343 harboring KRAS ${ }^{G 12 D}$ were treated with vehicle or SHP099 at $100 \mathrm{mg} / \mathrm{kg}$ as well as the MEK inhibitor Trametinib as a positive control, which was previously determined to have a significant antitumor effect in a similar PDX model (Supplementary Fig. 2c). SHP099 treatment resulted in a significant tumor growth inhibition similar to Trametinib compared to vehicle (Supplementary Fig. 3a). Treatment with SHP099 was also well tolerated with no obvious weight loss over the course of treatment (Supplementary Fig. 3b). These observations were associated with reduced levels of pERK, pMEK, and pAKT while the level of cleaved Caspase- 3 was increased in the treatment group (Supplementary Fig. 3c, d). Consistent with recent reports ${ }^{7-11}$, these results support the potential utility of SHP2 inhibitors in the treatment of mutant KRAS-driven PDAC.

Src phosphorylates and alters KRAS switch conformation. Phosphorylation of a protein alters the chemical environment and thus the chemical shifts of the modified residue, as well as spatially or sequentially adjacent residues. Ultimately, phosphorylation can induce conformational changes that affect protein function ${ }^{16}$. We first asked whether KRAS is phosphorylated by Src. In human embryonic kidney epithelial HEK293 cells transfected with plasmids encoding Src in combination with HAtagged HRAS, NRAS, or KRAS WT, pKRAS was detected at levels similar to pHRAS and pNRAS, which was abolished when lysates were treated with calf intestinal alkaline phosphatase (Fig. 1a). In contrast, another small GTPase protein, RAC2, exhibited negligible tyrosyl phosphorylation in the presence of Src (Supplementary Fig. 4a). Other tyrosine kinases, focal adhesion kinase (FAK) and SYK failed to phosphorylate KRAS while WT, but not catalytically impaired, Src effectively phosphorylated KRAS (Supplementary Fig. 4b). The ubiquitin ligase CBL, which has been shown to target Src for ubiquitin-mediated destruction ${ }^{17}$, had negligible effect on KRAS phosphorylation (Supplementary Fig. 4b). Furthermore, purified KRAS loaded with either GDP or GTP was phosphorylated to a similar extent by recombinant Src catalytic domain (Src ${ }^{\text {cat }}$ ) in vitro (Fig. 1b). These results suggest that KRAS is phosphorylated specifically via Src, which can be reversed by a phosphatase. 
a

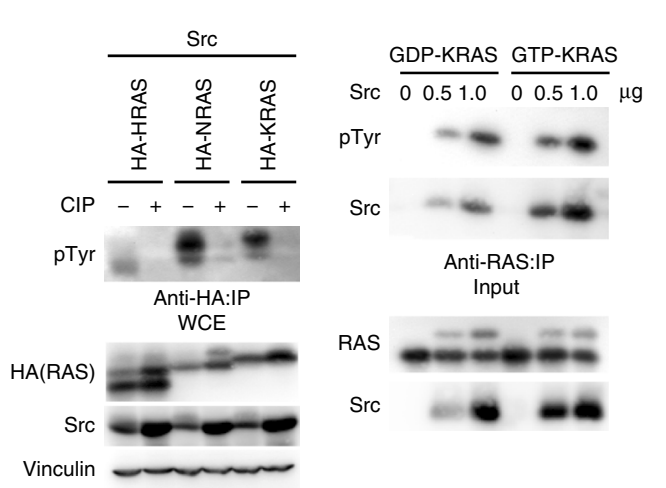

C

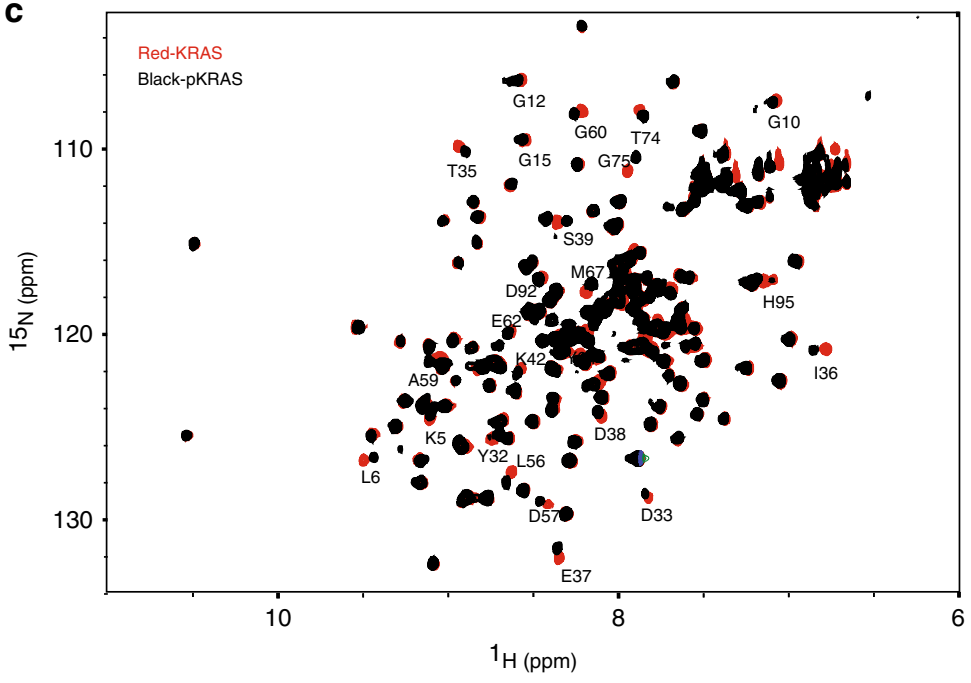

d

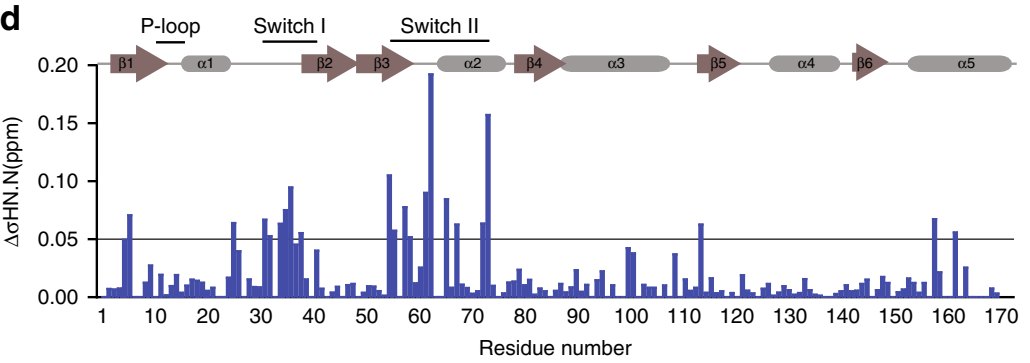

e

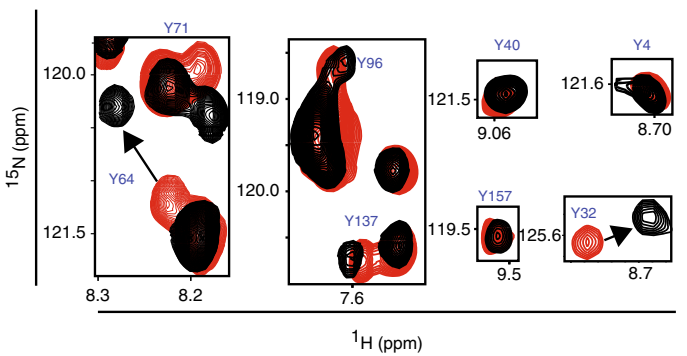

g
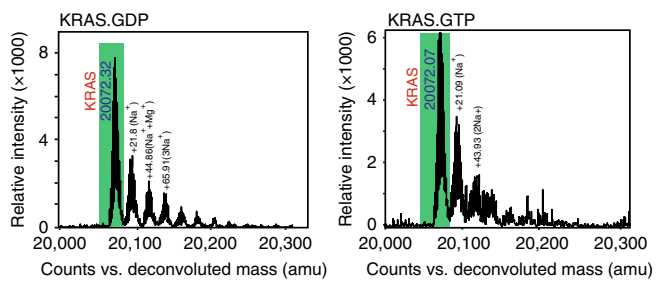

f

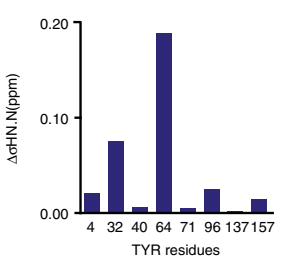

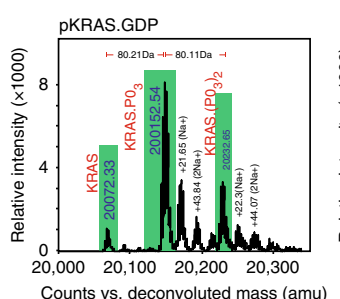

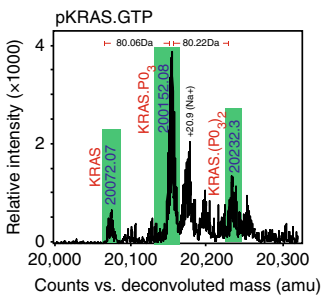

Fig. $1 \mathrm{Src}$ phosphorylation of KRAS alters the structure of the switch regions. a HEK293 cells transfected with the indicated plasmids were lysed, treated with $(+)$ or without $(-)$ calf intestinal alkaline phosphatase (CIP), immunoprecipitated, and immunoblotted with the indicated antibodies. $\mathbf{b}$ Purified recombinant human GDP- and GTP-bound KRAS protein were subjected to in vitro kinase assay using purified recombinant Src ${ }^{\text {cat }}$ kinase and were immunoprecipitated and immunoblotted with the indicated antibodies. c An overlay of $2 \mathrm{D}{ }^{1} \mathrm{H}-{ }^{15} \mathrm{~N}$ HSQC spectra of unmodified KRAS-GDP (red) and pKRAS-GDP phosphorylated by Src (black). The labeled residues are residues with notable chemical shift perturbation. $\mathbf{d}$ Histogram mapping the backbone $\mathrm{NH}$ chemical shift changes caused by Src phosphorylation. Chemical shift changes of the $\mathrm{N}$ and $\mathrm{NH}$ peaks of the unmodified vs. phosphorylated crosspeaks in the $2 \mathrm{D}^{1} \mathrm{H}-{ }^{15} \mathrm{~N} H S Q C$ spectra were calculated using the formula $\Delta \delta \mathrm{NH} . \mathrm{N}(\mathrm{ppm})=\sqrt{ }\left(\llbracket(\Delta \mathrm{H}) \rrbracket^{\wedge} 2+\llbracket(\Delta \mathrm{N} / 5) \rrbracket^{\wedge} 2\right)$ and plotted against residue number. e Overlay of ${ }^{1} \mathrm{H}-{ }^{15} \mathrm{~N}$ HSQC spectra of ${ }^{15} \mathrm{~N}$-KRAS tyrosine peaks before (red) and after (black) phosphorylation. The residue numbers are indicated in blue and the arrows indicate the direction of the chemical shift change. Full spectra are shown in $\mathbf{c}$. $\mathbf{f}$ Chemical shift changes of the KRAS tyrosine residues induced by phosphorylation. $\mathbf{g}$ Upper panel: Mass spectra of unmodified GDP-loaded KRAS (left) and KRAS loaded with the non-hydrolyzable GTP analog GMPPNP (right). The major peak of KRAS is shaded green, and the higher molecular weight peaks ( $+22 \mathrm{Da}$ ) correspond to sodium adducts (the predicted molecular mass of ${ }^{15} \mathrm{~N}$-KRAS (residues 1-173 C118S) is $20074.35 \mathrm{Da}$ ). Lower panel: Mass spectrum of GDP-loaded pKRAS (left) and GTP-loaded pKRAS (right) following treatment with Src in vitro. The three peaks highlighted in green correspond to unmodified KRAS, KRAS phosphorylated at one site $(+80 \mathrm{Da})$ and KRAS phosphorylated at two sites $(+160 \mathrm{Da})$. The immunoblot data are representative of at least three independent experiments

We next used NMR to examine potential conformational changes in KRAS induced by phosphorylation by examining chemical shift perturbations in the ${ }^{1} \mathrm{H}_{-}{ }^{15} \mathrm{~N}$ heteronuclear single quantum correlation (HSQC) spectra of isotopically labeled ${ }^{15} \mathrm{~N}$ KRAS-GDP in the presence of excess ATP upon addition of a small amount of catalytically active $\mathrm{Src}^{\text {cat }}$ (Src to KRAS molar ratio of 1:250) (Fig. 1c, d). Phosphorylation induced chemical shift perturbations on numerous KRAS residues, particularly those in the two switch regions, of magnitude consistent with Tyr phosphorylation $^{16}$. The most perturbed residues were Tyr32,
Asp33, Thr35, Ile36, and Glu37 in switch I and Leu56, Asp57, Ala59, Gly60, Tyr64, Met67, and Gly75 in switch II. Notably, Tyr32 and Tyr64 exhibited much larger chemical shift perturbations than the other six tyrosine residues in KRAS (Fig. 1e, f). The mass spectrum of intact KRAS following Src cat phosphorylation exhibited a major peak corresponding to singly phosphorylated KRAS (+80 Da), a smaller peak for doubly phosphorylated KRAS $(+160 \mathrm{Da})$ and a minor peak corresponding to unmodified KRAS, independent of the nucleotide bound (Fig. 1g). Other minor peaks in the mass spectra were assigned to sodium adducts. 
KRAS is phosphorylated via Src on conserved Tyr32 and Tyr64. To unambiguously identify the site(s) of phosphorylation, we conducted liquid chromatography (LC)-MS/MS sequencing of trypsin-digested KRAS following phosphorylation by Src. The LC-MS/MS data identified a tryptic peptide containing pTyr64 as the most abundant tyrosyl phosphorylated species, followed by one containing pTyr32, whereas the abundance of other phosphorylated peptides was negligible (Fig. 2a-c). Mass spectra of intact KRAS ${ }^{\mathrm{Y} 32 \mathrm{~F}}$ and KRAS ${ }^{\mathrm{Y} 64 \mathrm{~F}}$ mutants following Src ${ }^{\mathrm{cat}}$ phosphorylation each lacked doubly phosphorylated KRAS, while Y32F exhibited a major peak corresponding to singly phosphorylated KRAS and Y64F produced two peaks of similar intensity corresponding to un-phosphorylated and singly phosphorylated species (Fig. 2d). Consistent with these results, the cellular levels of tyrosyl phosphorylation of KRAS co-expressed in HEK293 cells with Src were reduced for Y32F and dramatically lower for the Y64F mutant in comparison to WT KRAS (Fig. 2e). We further separated and enriched the mono-phosphorylated and di-phosphorylated forms of KRAS via anion exchange chromatography (Supplementary Fig. 5) and observed using $\mathrm{Mn}^{2+}$-PhosTag sodium dodecyl sulfate-polyacrylamide gel electrophoresis (SDS-PAGE) ${ }^{18}$, two distinct slower migrating bands (i.e., mono- and di-phosphorylated), indicating that the modifications are tyrosyl phosphorylations catalyzed by Src (Fig. 2f). These results unambiguously identify Tyr32 and Tyr64 within switch I and II, respectively, as major phosphorylation acceptor sites on KRAS.

SHP2 dephosphorylates KRAS and promotes downstream signaling. We showed previously that SHP2 dephosphorylates pH/NRAS 6 and that alkaline phosphatase dephosphorylates Srcinduced tyrosyl pKRAS (Fig. 1a). Thus we asked whether SHP2 dephosphorylates pKRAS. Ectopic Flag-SHP2 expression was associated with decreased levels of Src-induced HA-pKRAS, together with increased levels of downstream pERK and pAKT signals in a dose-dependent manner (Fig. 3a). The constitutively active disease-causing Flag-SHP2 ${ }^{\mathrm{E} 76 \mathrm{~K}}$ mutant markedly reduced the level of pKRAS WT and G12V, while the catalytically dead SHP2 ${ }^{\mathrm{C} 459 \mathrm{~S}}$ mutant had a negligible effect (Fig. $3 \mathrm{~b}$ ). Further, we investigated the dephosphorylation of pKRAS in vitro using NMR and MS. Both approaches demonstrated that SHP2 dephosphorylates Src-phosphorylated KRAS (Fig. 3c-e). Notably, inhibition of SHP2 using 11a-1 attenuated SHP2-mediated dephosphorylation of pKRAS (Fig. 3f).

Endogenous RAS phosphorylation is regulated by Src and SHP2. We observed tyrosyl phosphorylation of endogenous RAS in WT mouse embryonic fibroblasts (MEFs) following plateletderived growth factor (PDGF) stimulation (Fig. 4a). Furthermore, Src/Fyn/Yes knockout (SYF-/-) MEFs showed negligible levels of endogenous pRAS while SHP2-/- MEFs displayed markedly enhanced levels of endogenous pRAS, which was further increased in the presence of PDGF treatment (Fig. 4a). The amount of RAS phosphorylation was inversely associated with downstream pERK and pAKT levels (Fig. 4a), supporting the notion that phosphorylation of RAS impairs downstream signaling. Similar results were observed in a reciprocal experiment in which the noted lysates were immunoprecipitated with anti-RAS antibody followed by anti-pTyr immunoblot (Fig. 4b). Importantly, inhibition of Src using the non-ATP competitive substrate pocket-directed Src inhibitor KX2-391 ${ }^{19}$ or Src-specific small interfering RNA (siRNA) led to increased levels of pERK in SHP2 $-/-$ MEFs (Supplementary Fig. 6a, b), suggesting that the observation of attenuated downstream of RAS signaling in SHP2 $-/$ MEFs is dependent, at least in part, on Src activity. The amplitude and duration of endogenous RAS phosphorylation as well as SHP2 and Src phosphorylation following growth factor stimulation were strongly and rapidly enhanced within $5 \mathrm{~min}$ (Fig. 4c). Notably, two slower migrating bands of endogenous RAS (consistent with di- and mono-phosphorylated forms as shown in Fig. 2f using purified KRAS) were detected by $\mathrm{Mn}^{2+}$-PhosTag SDS-PAGE in SHP2-/- MEFs, which were absent in SYF-/- MEFs (Fig. 4d). Similarly, SHP2-/- CFPAC1 harboring KRAS ${ }^{G 12 V}$ mutation showed markedly enhanced levels of tyrosyl-phosphorylated endogenous RAS in response to EGF (Fig. 4e). Lysates of PDAC tumors treated with SHP099 or vehicle only (Supplementary Fig. 3) were analyzed by $\mathrm{Mn}^{2+}$-PhosTag SDS-PAGE. The slower migrating RAS band was stronger in the SHP099 treatment group (Fig. 4f), suggesting that the inhibition of SHP2 via SHP099 curtailed dephosphorylation of pRAS in vivo. These results demonstrate that endogenous RAS is singly or doubly tyrosyl phosphorylated and that the phosphorylationmediated RAS GTPase cycle is a tightly controlled dynamic process.

Tyrosyl phosphorylation of KRAS stalls its GTPase cycle. To interrogate the impact of tyrosyl phosphorylation on the KRAS GTPase cycle, we used a real-time NMR GTPase assay ${ }^{20,21}$ to measure nucleotide exchange and hydrolysis rates of unmodified KRAS and pKRAS. For the exchange assay, GDP-loaded ${ }^{15} \mathrm{~N}$ KRAS or pKRAS were mixed with a ten-fold molar excess of GTP $\gamma$ S in an NMR tube, and consecutive ${ }^{1} \mathrm{H}^{-15} \mathrm{~N}$ HSQC spectra were acquired as the exchange reaction proceeded. The intensities of peaks from residues whose chemical shifts are sensitive to GDP/GTP binding were used to calculate the rate of the nucleotide exchange (Fig. 5a). Interestingly, phosphorylation of KRAS increased the intrinsic rate of nucleotide exchange by $~ 2.6-$ fold, while reducing the sensitivity of pKRAS to the exchange activity of the catalytic domain of the GEF SOS1 (SOScat) ${ }^{22}$ (Fig. 5b, c and Supplementary Table 1a). Specifically, the addition of recombinant SOScat to unmodified KRAS at a ratio of 1:600 accelerated the exchange reaction 17 -fold, whereas nucleotide exchange of pKRAS was enhanced by <3-fold (Fig. 5b, $c$ and Supplementary Table 1a). The activation of KRASG12V mutant by SOS was similarly impaired upon phosphorylation (Supplementary Fig. 7a). Thus, while phosphorylation of KRAS by Src mildly increases the intrinsic nucleotide exchange rate, these posttranslational modifications impair its activation by SOS by almost an order of magnitude.

We next loaded KRAS or pKRAS with GTP and monitored its hydrolysis by real-time NMR. pKRAS exhibited an intrinsic GTP hydrolysis rate that was $\sim 3$-fold lower than that of unmodified KRAS (Fig. 5d, e and Supplementary Table 1b). In the presence of 1:3000 molar ratio of recombinant GAP domain of RASA1, the rate of GTP hydrolysis of unmodified KRAS was accelerated by $>300 \%$ while that of pKRAS increased by $<20 \%$ (Fig. $5 \mathrm{~d}$, e and Supplementary Table 1b). KRAS ${ }^{G 12 \mathrm{~V}}$ mutant has, however, impaired intrinsic hydrolysis and is insensitive to GAP stimulation, thus phosphorylation had little impact on these rates (Supplementary Fig. 7b). These results indicate that phosphorylation of KRAS alters its intrinsic nucleotide exchange and hydrolysis rates in a manner that favors accumulation of pKRASGTP, while simultaneously rendering it resistant to inactivation by GAP activity and largely insensitive to GEF activity, thus uncoupling pKRAS from upstream regulation.

Tyrosyl phosphorylation of KRAS impairs effector RAF binding. We asked whether phosphorylation of KRAS via Src influenced its binding to effector proteins. Tyrosyl phosphorylation of purified GTP-loaded KRAS via Src markedly attenuated 
a

MTEYKLVVVGAGGVGK GEGFLCVFAINNTKSFEDIHHYREQIKRVKDSEDVPMVLVGNKCDLPSRTVDTKQAQDLARSYGIPFIETSAKTRQ RVEDAFYTLVREIRQYRLKKISKEEKTPGCVKIKKCIIM

b Peptide: (K)SALTIQLIQNHFVDEpYDPTIEDSYR

KRAS phosphotyrosine residue $\mathrm{pY} 32$

Precursor ion $m / z 1027.44[\mathrm{M}+3 \mathrm{H}]^{3+}$

Spectral count: 15
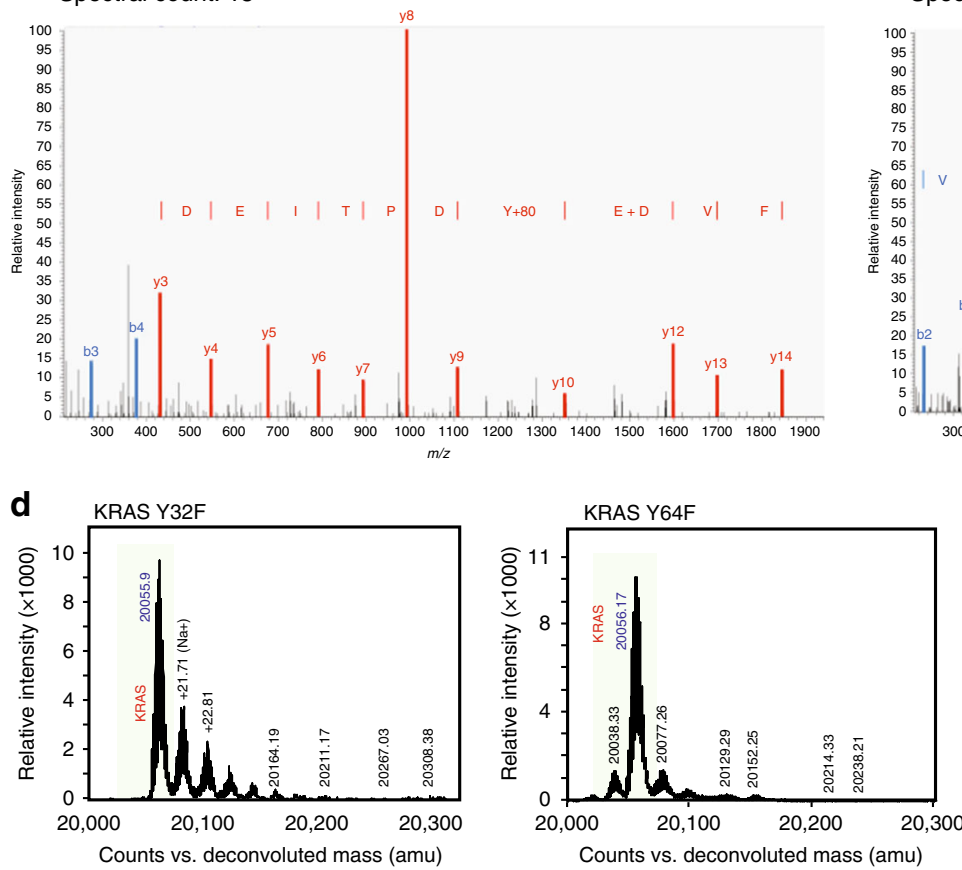

pKRAS Y32F
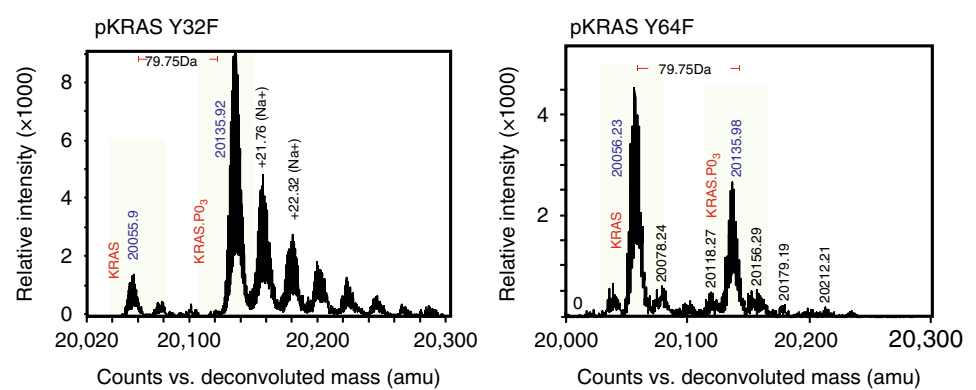

C Peptide: (K)QVVIDGETCLLDILDTAGQEEpYSAMR

KRAS Phosphotyrosine residue pY64

Precursor ion $\mathrm{m} / \mathrm{z} 1013.09[\mathrm{M}+3 \mathrm{H}]^{3+}$

Spectral count: 250

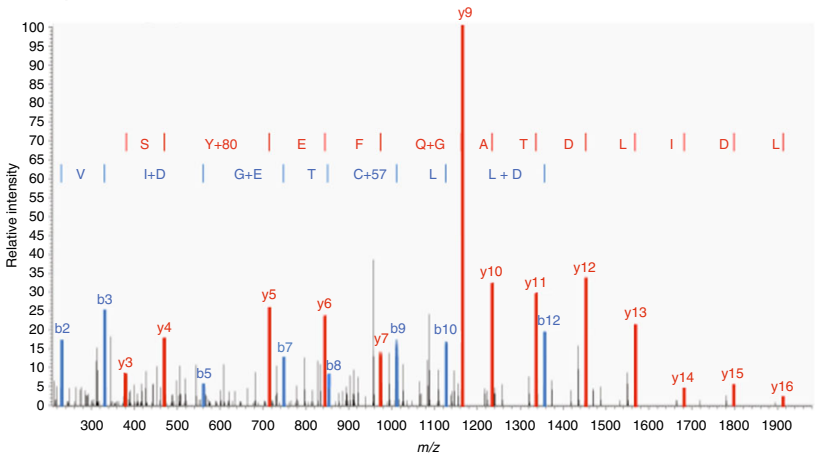

e

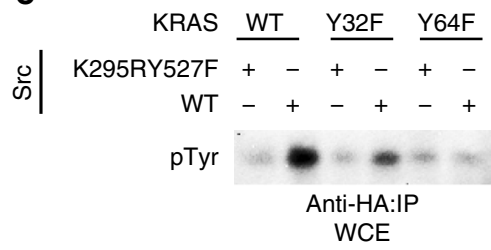

HA (KRAS)

Src

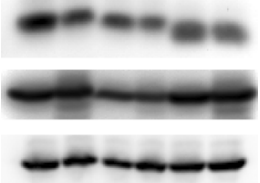

f

$\mathrm{Mn}^{2+}-$ Phos-tag

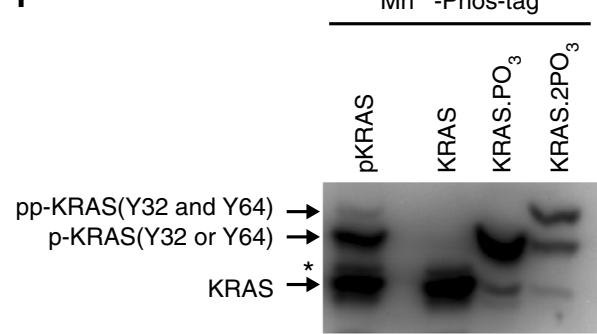

No $\mathrm{Mn}^{2+}$-Phos-tag

Fig. 2 Src phosphorylates KRAS tyrosine 32 and 64. a-c Two phosphorylated tyrosine residues from the KRAS protein sequence (a) were identified from in vitro kinase reaction samples that were reduced/alkylated, trypsin-digested, and analyzed by LC-MS. MS/MS spectra matched indicated KRAS tryptic peptides containing residue Y32 (b) and Y64 (c). d Upper panel: Mass spectra of unmodified KRASY32F (left) and KRASY64F (right). Lower panel: Mass spectrum of pKRAS ${ }^{Y 32 F}$ (left) and pKRASY64F (right). The major peak of KRAS is shaded light green. e HEK293 cells were transfected with the indicated plasmids. Cells were lysed, immunoprecipitated, and immunoblotted with the indicated antibodies. The immunoblot data are representative of at least three separate experiments. f GTP-bound KRAS were subjected to in vitro kinase assay using purified Src kinase (left lane) and were separated into unphosphorylated (KRAS), mono-phosphorylated $\left(K R A S . \mathrm{PO}_{3}\right)$, and di-phosphorylated $\left(\mathrm{KRAS} .2 \mathrm{PO}_{3}\right)$ forms by anion exchange chromatography. These samples were then analyzed by $50 \mu \mathrm{M} \mathrm{Mn}{ }^{2+}$-PhosTag SDS-PAGE (upper panel) or conventional SDS-PAGE (lower panel) and immunoblotted with antiRAS antibody. Asterisk indicates non-specific band. IP immunoprecipitation, WCE whole-cell extract. The immunoblot data are representative of at least three independent experiments 
a
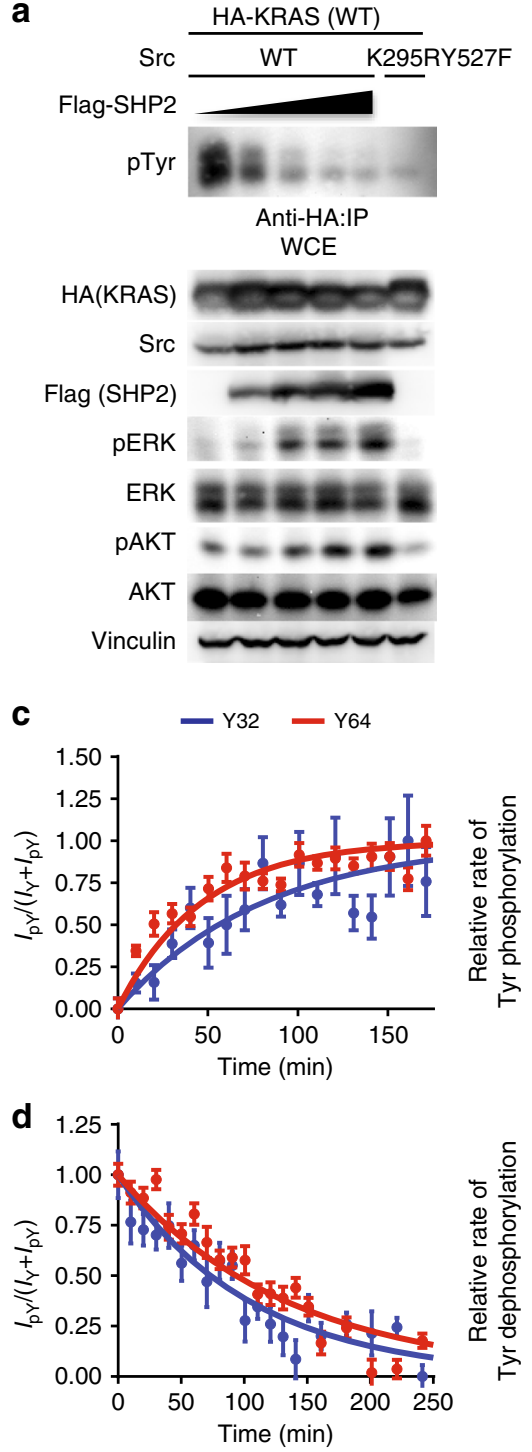

b
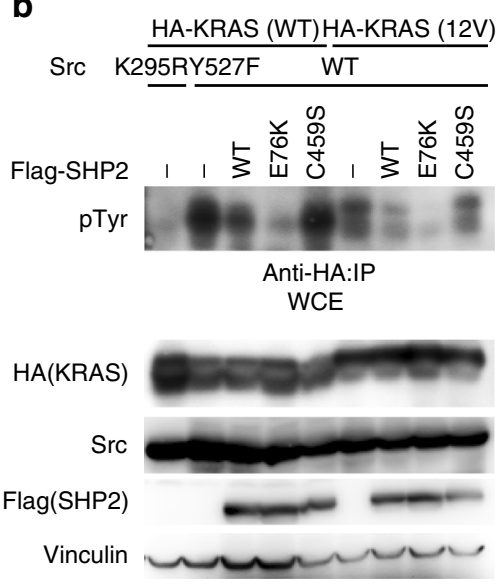

f

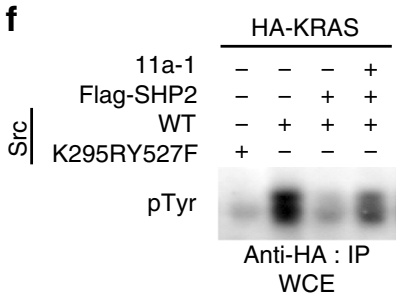

HA (KRAS)

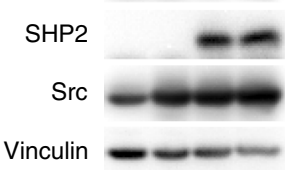

e

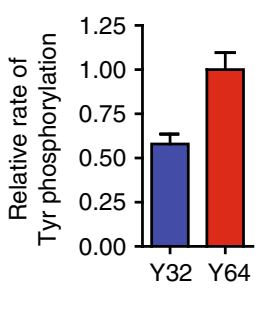

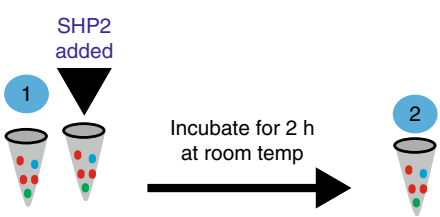
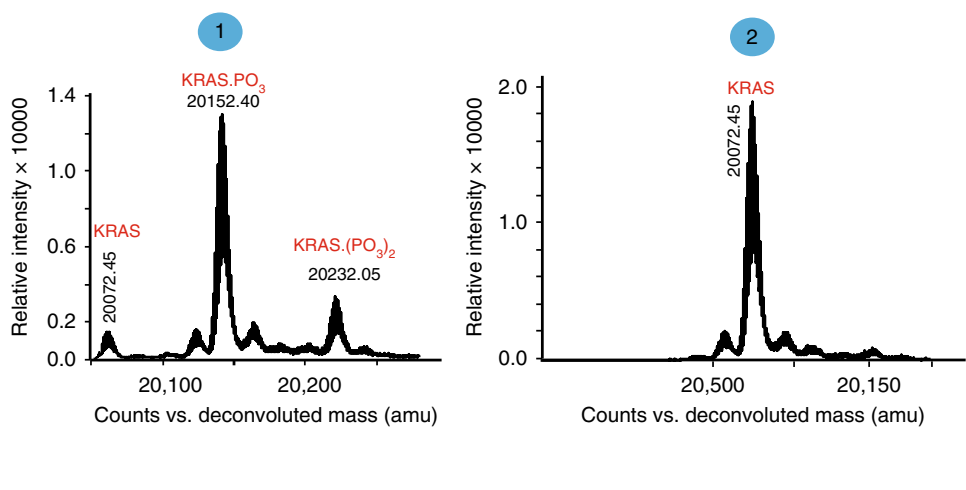

Fig. 3 SHP2 dephosphorylates tyrosyl phosphorylated KRAS. a, b HEK293 cells were transfected with the indicated plasmids. Cells were lysed, immunoprecipitated, and immunoblotted with the indicated antibodies. c Phosphorylation was initiated by addition of $2 \mu \mathrm{M}$ of $\operatorname{Src}$ to $250 \mu \mathrm{M}$ of KRAS in the presence of $2 \mathrm{mM}$ ATP and $1 \mathrm{mM}$ activated sodium vanadate. Sequential ${ }^{1} \mathrm{H}-{ }^{15} \mathrm{~N}$ HSQC NMR spectra were collected, and peaks from un-phosphorylated and phosphorylated Tyr32 (blue) and Tyr64 (red) were integrated from each spectrum to plot curves of the fraction phosphorylated vs. time for each site. The error bars correspond to the noise-to-signal ratio of the respective peaks. The relative rates of phosphorylation of each site determined from a representative experiment (performed in duplicate) by curve fitting are shown in histograms on the right. The error bars indicate the standard curve fitting error obtained from the fitting analysis. d SHP2 $(2 \mu \mathrm{M})$ was added to Src-phosphorylated KRAS sample purified in the presence of vanadate, and sequential ${ }^{1} \mathrm{H}-{ }^{15} \mathrm{~N}$ NMR spectra were collected to monitor dephosphorylation. Similar to c, the fractions of phosphorylated Tyr32 (blue) and Tyr64 (red) were plotted and rates of dephosphorylation were determined and are shown in the histograms. Rate curves from a representative experiment (performed in duplicate) were fitted to one phase exponential decay/association functions using the GraphPad Prism 4.0 software. Histogram error bars indicate the standard curve fitting error obtained from the fitting analysis. e Top panel; experimental set-up whereby two tubes containing $200 \mu \mathrm{M}$ of KRAS and $3 \mu \mathrm{M}$ of Src were incubated in in the presence of $2 \mathrm{mM} \mathrm{ATP}$ for $2 \mathrm{~h}$ (in the absence of vanadate). Sample 1 was then snap frozen and stored at $-80^{\circ} \mathrm{C}$ for $\mathrm{MS}$ analysis, and $3 \mu \mathrm{M}$ of SHP2 was added to the remaining tube and incubated for an additional $2 \mathrm{~h}$. Sample 2 was then frozen. Bottom panels; MS spectra of samples 1 and 2 with phosphorylation state of each mass indicated. $\mathbf{f H E K} 293$ cells transfected with the indicated plasmids were treated with ( + ) or without $(-) 10 \mu \mathrm{M}$ of $11 a-1$, lysed, immunoprecipitated, and immunoblotted with the indicated antibodies. The immunoblot data are representative of at least three independent experiments

the interaction with RAF-RBD in pull-down assays (Fig. 6a). To quantify the effect of phosphorylation on this interaction, we performed biolayer interferometry using un-phosphorylated, mono-phosphorylated, and di-phosphorylated KRAS, which were separated and enriched via anion exchange chromatography (Supplementary Fig. 5). Un-phosphorylated KRAS bound with a $K_{\mathrm{D}}$ value of $\sim 300 \mathrm{nM}$, whereas the mono-phosphorylated fraction, which contains a mixture of pTyr32 and pTyr64 KRAS, exhibited $\sim 2$-fold weaker binding, and di-phosphorylated KRAS exhibited severely impaired binding to BRAF-RBD and much higher concentrations were required to detect its binding (Fig. 6b and Supplementary Fig. $8 \mathrm{a}-\mathrm{c}$ ). The high protein concentrations promoted some non-specific interactions that reduced the accuracy of $K_{\mathrm{D}}$ determination; however, di-phosphorylation of KRAS reduced its affinity for BRAF by at least 15 -fold. Notably, the oncogenic KRAS ${ }^{\mathrm{G} 12 \mathrm{~V}}$ and KRAS ${ }^{\mathrm{G} 12 \mathrm{D}}$ mutants were also 
a

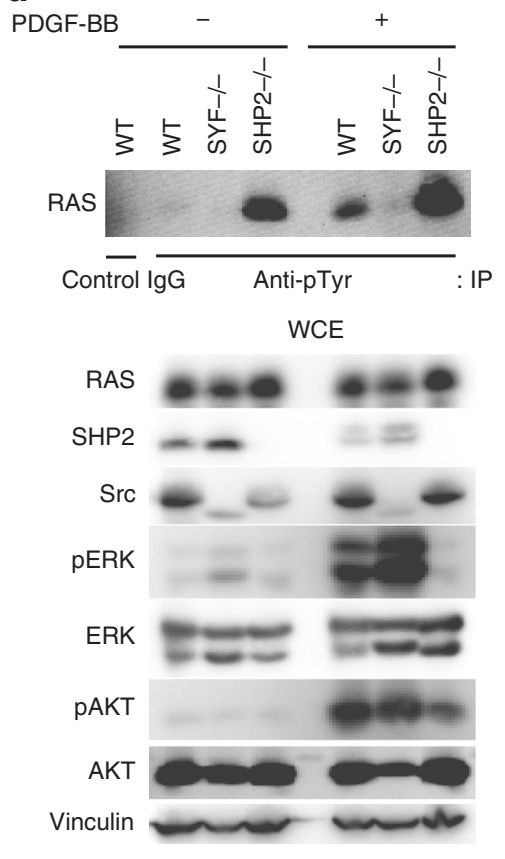

b
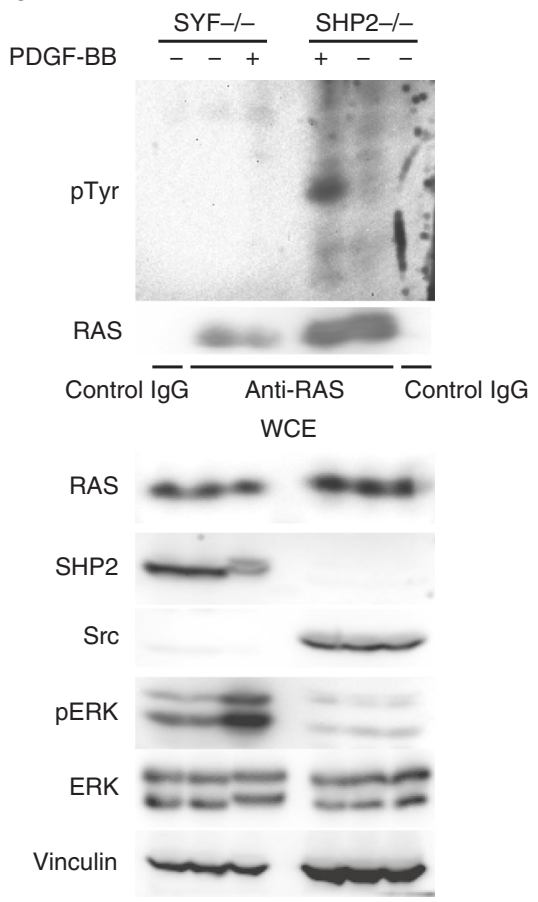

e

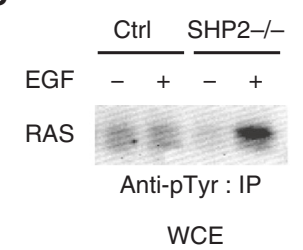

RAS

SHP2

pERK

ERK

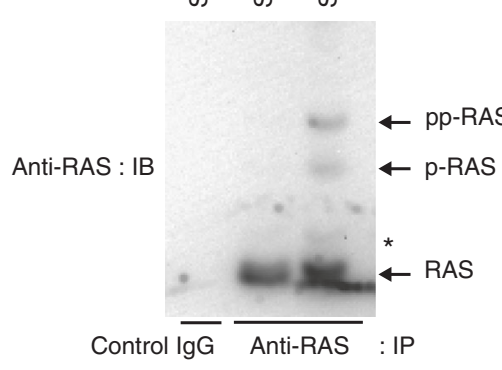

d
C

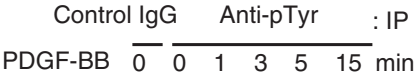

RAS

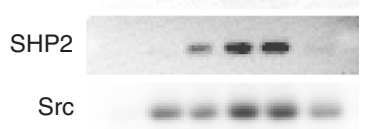

WCE

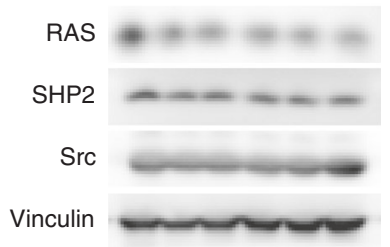

Vinculin

WCE

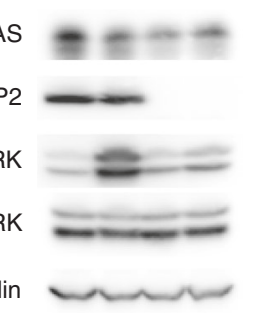

f

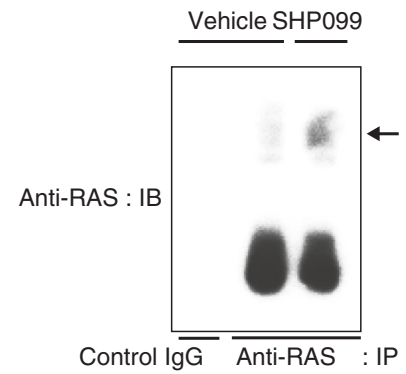

Fig. 4 Endogenous RAS phosphorylation is regulated temporally by Src and SHP2. a WT, Src/Yes/Fyn (SYF) -/- and CRISPR/Cas9-mediated SHP2-/MEFs were serum starved and treated with $20 \mathrm{ng} / \mathrm{ml}$ of PDGF-BB. Equal amounts of lysates were immunoprecipitated with isotype-matched antibody or anti-pTyr antibody and immunoblotted with the indicated antibodies. b SYF-/- and SHP2-/-MEFs were serum starved and treated with $20 \mathrm{ng} / \mathrm{ml}$ of PDGF-BB. Equal amounts of lysates were immunoprecipitated with isotype matched antibody or anti-RAS antibody and immunoblotted with the indicated antibodies. c MEFs were serum starved and treated with $20 \mathrm{ng} / \mathrm{ml}$ of PDGF-BB or were left untreated for the indicated periods of time. Equal amounts of lysates were immunoprecipitated with isotype-matched antibody or anti-pTyr antibody and immunoblotted with the indicated antibodies. $\mathbf{d}$ SYF-/- and SHP2-/- MEF lysates were immunoprecipitated with isotype-matched antibody or anti-RAS antibody and analyzed by $50 \mu \mathrm{M} \mathrm{Mn}{ }^{2+}-\mathrm{Phos} T a g$ SDS-PAGE. Asterisk indicates non-specific band. e CRISPR/Cas9-mediated non-target (Ctrl) or SHP2-/- CFPAC1 cells were serum starved and treated with ( + ) or without (-) $10 \mathrm{ng} / \mathrm{ml}$ of EGF. Equal amounts of lysates were immunoprecipitated and immunoblotted with the indicated antibodies. $\mathbf{f}$ OCIP.343 xenograft tumor lysates from the indicated treatment groups were immunoprecipitated with isotype-matched antibody or anti-RAS antibody and analyzed by

$\mathrm{Mn}^{2+}$-PhosTag SDS-PAGE. Arrow indicates a slower migrating band. The immunoblot data are representative of at least three independent experiments

phosphorylated by Src in a dose-dependent manner, which was associated with markedly attenuated RAF-RBD binding (Fig. 6b, c). Moreover, the dephosphorylation status of KRAS WT or $12 \mathrm{~V}$ was associated with enhanced binding to RAS-binding domain (RBD) of RAF (Fig. 6d). These results demonstrate that phosphorylation of WT or oncogenic KRAS mutants attenuates its binding to effector RAF.

Moreover, our finding that pKRAS has impaired affinity for RAF-RBD has an important implication for the most widely used assay of RAS activation (i.e., the RBD pull-down assay). It has been consistently reported that inhibition of SHP2 reduces the amount of RAS-GTP pulled down in such assay, which has been interpreted as reduced RAS activation due to impaired SOS activation or increased GAP activity. However, our results demonstrate that phosphorylation of RAS-GTP upon SHP2 inhibition can also contribute to reducing RAS binding to RBD.

\section{Discussion}

SHP2 is a well-established major regulator of RAS-to-RAF-toMAPK signal pathway and somatic gain-of-function SHP2 
a

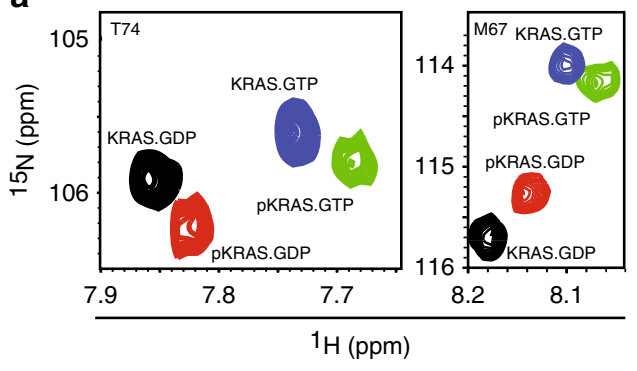

b

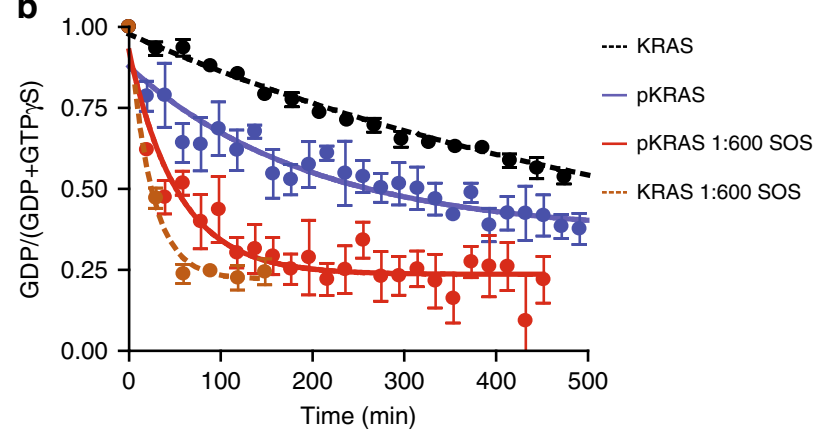

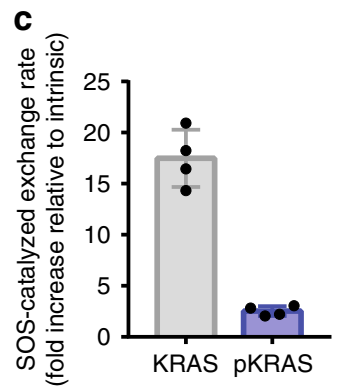

d

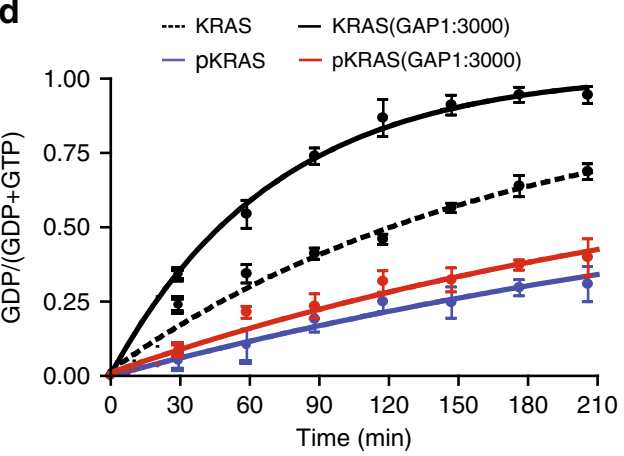

e

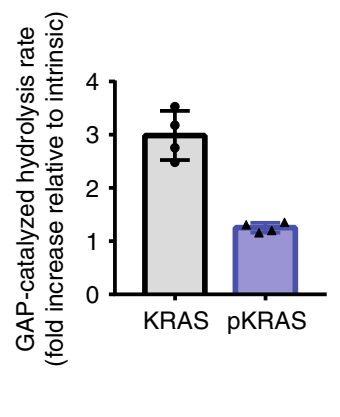

Fig. 5 Tyrosyl phosphorylation of KRAS disrupts GTPase cycle. a Overlay of four ${ }^{1} \mathrm{H}-{ }^{15} \mathrm{~N}$ HSQC spectra of two representative residues T74 and M67, in which the four peaks represent GDP-loaded KRAS (black), GTP-loaded KRAS (blue), GDP-loaded Src-phosphorylated (p)KRAS (red), and GTP-loaded pKRAS (green). b Real-time NMR-derived nucleotide exchange curves for unmodified vs. Src-phosphorylated KRAS. A 250 $\mu M$ sample of GDP-loaded ${ }^{15} \mathrm{~N}$ KRAS (black) or pKRAS (blue) was incubated with ten-fold molar excess of GTP $\gamma$ S. Each dot represents mean fraction of KRAS that is loaded with GDP on the basis of peak intensities $\left[I_{G D P} /\left(I_{\text {GDP }}+I_{\text {GTPYS }}\right)\right]$ from the same three residues of KRAS and pKRAS. SOScat was added at a ratio of 1:600 to KRAS (orange) or pKRAS (red). c Src phosphorylation of KRAS impairs SOScat-assisted nucleotide exchange. Fold increase in the rate of exchange upon addition of SOScat to KRAS or pKRAS. The reaction was performed twice in the absence and twice in the presence of SOScat and four ratios were determined from each pairwise comparison. d GTP hydrolysis curves illustrating intrinsic and RASA1 GAP domain (1:3000 ratio) assisted GTP hydrolysis for unmodified KRAS and pKRAS. e Src phosphorylation of KRAS reduces sensitivity to GAP activity. Fold increase in the rate of GTP hydrolysis with addition of RASA1 GAP domain for KRAS vs. pKRAS. The reaction was performed twice in the absence and twice in the presence of RASA1 GAP domain and four ratios were determined from each pairwise comparison. $\mathbf{b}$, $\mathbf{d}$ present a single representative experiment that was repeated at least twice. Error bars represent the standard deviation of the fraction GDP as reported by three pairs of cross-peaks. Error bars in $\mathbf{c}$, $\mathbf{e}$ indicate standard deviation of the fold changes

mutations or overexpression have also been identified in several hematologic malignancies as well as solid tumors such as gastric, breast, lung and pancreatic cancers and RASopathies ${ }^{1,2,6,23-25}$. These observations have led to the notion that SHP2 could be used as prognostic marker and potentially targeted for therapy even prior to our reporting of its direct involvement in the regulation of RAS ${ }^{6}$. Although we and others have shown that the treatment of RAS-dependent cancers can benefit from SHP2 inhibition $^{6-11}$, the molecular mechanisms that relate SHP2 to RAS signaling have remained unclear and controversial ${ }^{10}$.

SHP2 is a phosphatase containing two $\mathrm{SH} 2$ domains, a catalytic PTP domain and a C-terminal regulatory tail with sites of tyrosyl phosphorylation. At basal state, SHP2 adopts a closed inactive conformation in which its SH2 and PTP domains are occluded ${ }^{26}$. In the activated open state, the PTP domain of SHP2 is exposed to substrates and the $\mathrm{SH} 2$ domains are available to interact with pTyr residues. Phosphorylated SHP2 can recruit GRB2 and SOS to phosphorylated receptor tyrosine kinases, and the docking of SHP2 SH2 domains to substrates may promote the catalytic function of the PTP domain ${ }^{27,28}$. Several lines of evidence suggest the importance of the catalytic function of SHP2 in regard to its role for RAS activation, although the relevant targets are still debated $^{29}$. SHP2 has been implicated as a regulator of RAS GTPase cycle by regulating GEF and GAP localization by dephosphorylating the p120 RASGAP docking sites on EGFR and GAB1 $1^{30,31}$ or GRB2-binding sites on Sprouty, reversing its negative regulation of GRB2/SOS recruitment ${ }^{32}$. These models, which are not necessarily mutually exclusive with the presented model, involve SHP2 regulation of the RAS GTPase cycle by modulation of the regulators of RAS.

Although the biological significance remains unclear, phosphorylation of the residues homologous to Tyr32 on RHOA (Tyr34), RAB24 (Tyr37), and RAN (Tyr39) has been described $33-35$ and Src has also been shown to phosphorylate CDC42, R-RAS, and the heterotrimeric $G$ protein a subunit ${ }^{36-38}$. There are also examples of phosphorylation affecting the molecular switch function of RAS. For example, HRAS Tyr137 phosphorylation by Abelson tyrosine protein kinase has been shown to allosterically enhance the binding of HRAS to effector RAF ${ }^{39}$ while phosphorylation of a plant GTPase Toc34 is thought to affect its GTPase activity 40,41 . Here we show that endogenous RAS is tyrosyl phosphorylated and that KRAS is phosphorylated by Src on Tyr32 and Tyr64 in a kinase- and site-specific manner. We show that tyrosyl phosphorylation of KRAS alters the conformation of switch I and II, consequently attenuating GEF-mediated nucleotide exchange, GAP-assisted GTP hydrolysis, and the binding affinity of KRAS to the effector RAF (Fig. 7a). These results demonstrate that phosphorylation of KRAS stalls the GTPase cycle into a "dark state" while SHP2-mediated dephosphorylation is required to maintain dynamic cycling or to unleash signaling-competent KRAS from the dark state of pKRAS. Importantly, KRAS mutations frequently occur at codon 12 and substitution mutations encoding G12D and 


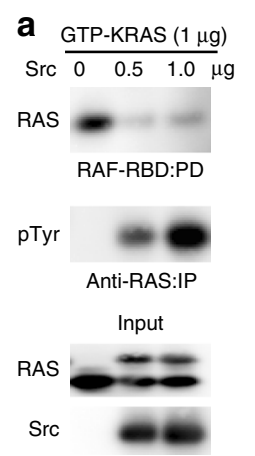

C

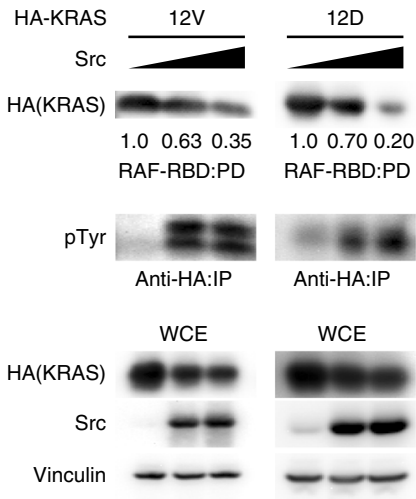

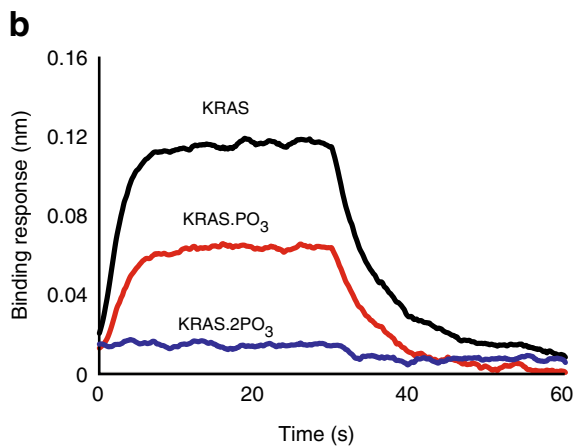
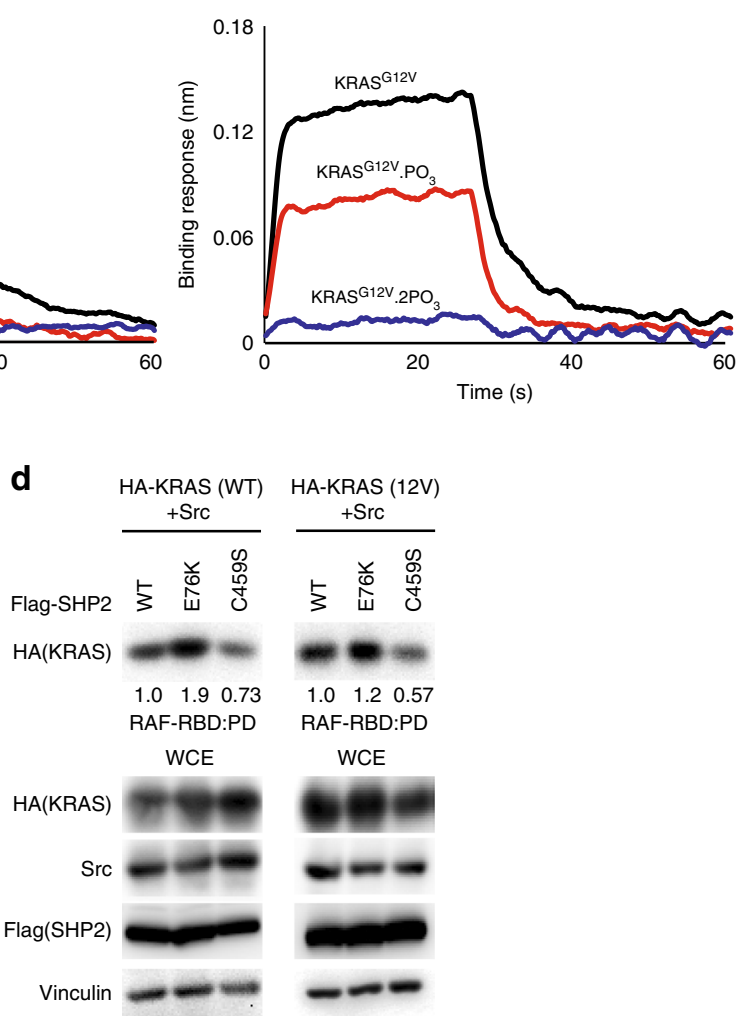

Fig. 6 Tyrosyl phosphorylation of KRAS attenuates binding to RAF. a Purified recombinant human GTP-bound KRAS protein was subjected to in vitro kinase assay using purified recombinant Src kinase and pulled down using Raf-1:RBD-conjugated beads or immunoprecipitated and immunoblotted with the indicated antibodies. b The binding of un-, mono-, and di-phosphorylated KRAS, each at a concentration of 250 nM, to immobilized GST-BRAF-RBD was measured using biolayer interferometry (Octet). The full binding curves at a range of KRAS concentrations is shown in Supplementary Fig. 8. Left panel: wild-type KRAS. Right panel: KRASG12V mutant. c, d HEK293 cells were transfected with the indicated plasmids. Cells were lysed, pulled down using Raf-1: RBD-conjugated beads, or were immunoprecipitated and immunoblotted with the indicated antibodies. Numerical values indicate the ratio of quantified signals over the control (lane 1 of each panel; set at 1.0) determined by densitometry using the Image J software. The immunoblot data are representative of at least three independent experiments. PD pulldown

G12V are among the most prevalent in PDAC tumors ${ }^{2,42}$. Notably, we show that Src- and SHP2-mediated regulation of KRAS extends to these common KRAS mutants and that the inhibition of SHP2 activity disrupts the phosphorylation cycle, shifting the equilibrium of oncogenic KRAS toward the dark state (Fig. 7a). Although it is formally unknown the stoichiometry of KRAS phosphorylation in cells, it appears reasonable that dynamic (de)phosphorylation of KRAS in the appropriate spatiotemporal context upon external stimulus would be sufficient to trigger downstream signaling. Src and SHP2 both have well-established functions at receptor tyrosine kinases and are thus poised to regulate the phosphorylation of the local KRAS that is most relevant to signaling (i.e., KRAS with the potential to become activated by SOS at activated receptors).

A crystal structure of RAS in complex with SOS reveals that both Tyr32 and Tyr64 form points of contact with SOS. The mechanism underlying the GEF activity of SOS involves the insertion of a helix from the Cdc25 domain of SOS into the RAS nucleotide-binding domain to open up the two switch regions to destabilize nucleotide binding. This helical interface includes a direct interaction between Tyr32 and Asn944 of SOS (PDB: 1BKD), and the Tyr32 hydroxyl forms a hydrogen bond with Ser39 in switch I and a water molecule, which appears to stabilize the open conformation of RAS ${ }^{43}$ (Fig. 7b). Meanwhile, Tyr64 inserts into a hydrophobic pocket on SOS where its hydroxyl forms a hydrogen bond with the backbone of Gly931 (Fig. 7b). Mutations of either Tyr32 or Tyr64 have been shown to disrupt interaction with SOS and impair the sensitivity of RAS to its catalytic activity ${ }^{44,45}$, suggesting that the reduced sensitivity of pKRAS to SOS is likely due to disruption of interactions with either Tyr hydroxyl in combination with steric hindrance and unfavorable electrostatics of the phosphate group.

Intrinsic GTP hydrolysis by RAS involves attack on the $\gamma$ phosphate by a catalytic water molecule 46,47 , and RAS mutations that perturb the activation of the catalytic water molecule reduce the intrinsic hydrolysis rate ${ }^{48-50}$. This water molecule interacts with the $\gamma$-phosphate of GTP, which is stabilized by a second "bridging" water molecule that interacts directly with the sidechain hydroxyl of Tyr32 (Fig. 7c), suggesting that this coordination would likely be spoiled by the addition of a phosphate moiety to the Tyr32 hydroxyl. As the same water molecule participates in GAP-catalyzed GTP hydrolysis, the effect of phosphorylation on its coordination may partially explain the reduced sensitivity to GAP activity. Further, the crystal structure of RAS in complex with the GAP domain of RASA1 revealed extensive interaction with both switch I and II, and in particular Tyr64, which forms a hydrophobic interface between RAS and the GAP domain $^{51}$ (Fig. 7d). Moreover, anionic substitutions of Tyr64 (Y64D/E) disrupt GAP-assisted hydrolysis ${ }^{52,53}$, supporting the notion that Tyr64 phosphorylation would impair interaction with the GAP.

Another important aspect of KRAS phosphorylation is that tyrosyl phosphorylation of KRAS at Tyr32 and Tyr64 reduces the affinity to RAF, which renders direct shut down of KRASmediated MAPK pathway. The crystal structure of RAS in 


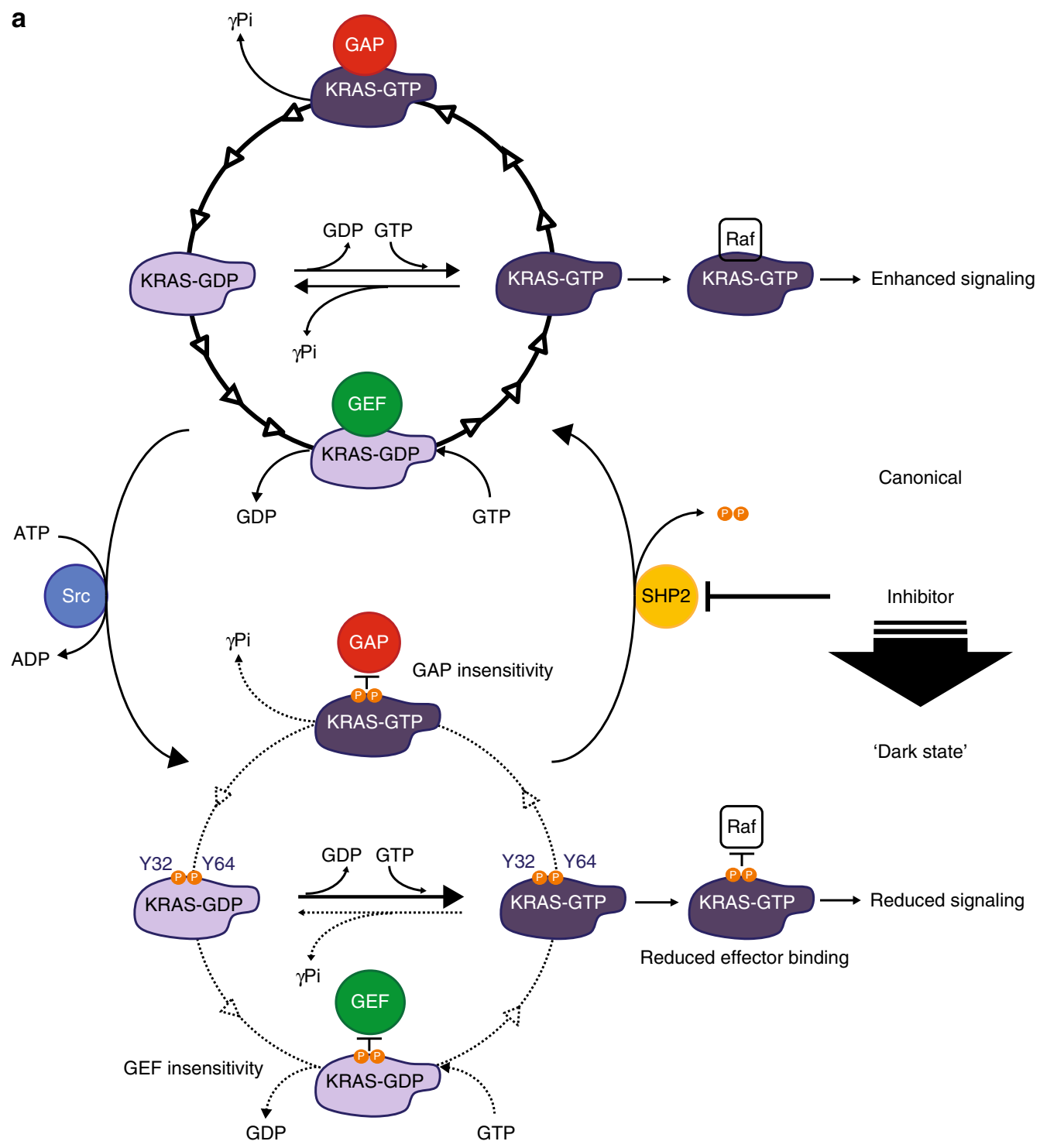

b

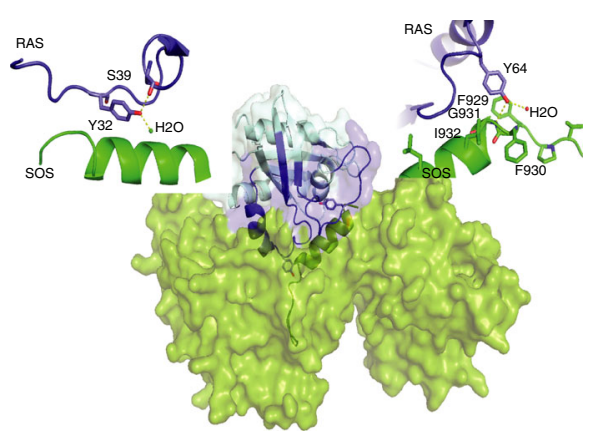

C

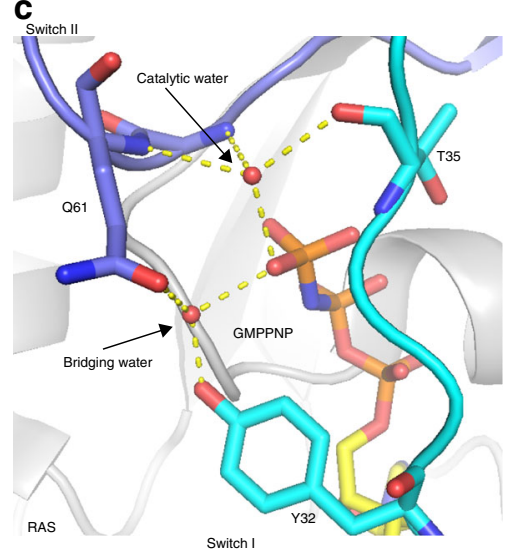

d
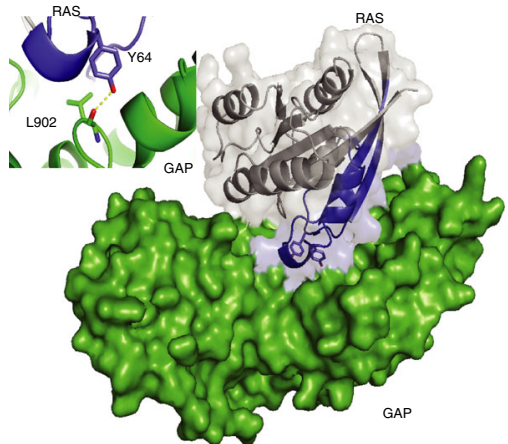

complex with RAF demonstrates that switch I forms the core of the binding site ${ }^{54}$. Notably, Tyr32 is found in the middle of the RAS effector-binding site, suggesting how phosphorylation of this residue reduces the affinity to RAF, thereby attenuating the downstream RAF-to-MEK-to-ERK pathway. However, considering that both switch I and II regions are critical for binding multiple effector proteins, discerning the relative contribution of Tyr32 and/or Tyr64 modification on individual effector recruitment will be important for understanding how RAS phosphorylation governs the signaling of a myriad of pathways.

Here we show that Src phosphorylation decouples KRAS from regulation by GEFs and GAPs, leading to the accumulation of a 
Fig. 7 Model of the impact of phosphorylation on KRAS GTPase cycle and structural rationale. a Phosphorylation decouples KRAS from effectors and GTPase cycle regulation. The GTPase cycle of unmodified KRAS is shown on top. Nucleotide exchange and hydrolysis are accelerated by the activities of GEFs and GAPs, respectively. GTP-loaded KRAS binds and activates RAF. Src phosphorylation of Tyr32 and Tyr64 of KRAS-GDP or KRAS-GTP alters all steps in this GTPase cycle (lower part). Upon phosphorylation, KRAS becomes insensitive to regulation by both GEFs and GAPs, whereas intrinsic nucleotide exchange is enhanced and intrinsic GTP hydrolysis is impaired. This leads to the accumulation of phosphorylated KRAS-GTP, which has reduced affinity for RAF, thus phosphorylation limits KRAS signaling. SHP2 dephosphorylates KRAS and thereby unleashes KRAS-GTP to rapidly activate RAF. Inhibition of SHP2 promotes accumulation of phosphorylated KRAS, dampening RAF signaling and subsequently suppressing oncogenesis. Line thickness and number of arrowheads represent relative reaction rates of each step. $\mathbf{b}$ Ribbon diagram SOS (green) contact with Tyr32 and Tyr64 of RAS (PDB ID 1BKD). c Proposed coordination of switch I and switch II RAS residues in intrinsic GTP hydrolysis (PDB ID 4GON). d Switch I and II (blue) of RAS are the main interaction site with GAP (green) (PDB ID 1WQ1)

GTP-bound "dark state" that is impaired in its ability to engage and activate RAF kinase. While a large body of work has established pro-oncogenic roles for Src in tumor development and metastasis, its phosphorylation of RAS is distinctively a tumorsuppressive function. Although preclinical studies implicated Src as a therapeutic target for cancer, phase II trials failed to demonstrate a significant clinical benefit of Src inhibitor monotherapy for metastatic solid tumors including breast, prostate, head and neck, colon, pancreatic, or non-small cell lung cancers $^{55}$. These clinical trials, however, were performed in unselected patients, lacking effective response biomarkers to guide the design of clinical trials, which was partly due to the enormous complexity of Src signaling. Recent preclinical data suggest that cells with elevated Src activity are more likely to respond to Src inhibition, while tumors with diminished Src signaling resulting from alternative oncogenic pathways may contribute to de novo resistance to Src inhibitors ${ }^{56-59}$. These, albeit limited, studies demonstrate that hyperactive Src signaling may potentially serve as a biomarker for successful targeting of Src and clinical efficacy. However, other molecular alterations in cancers may impact on the response of cancer cells to Src inhibitors. For example, c-MET amplification in gastric cancer as well as the autophagy pathway were shown to promote resistance to Src inhibitors ${ }^{59,60}$ while acquired resistance of ER+ breast cancers to the Src family kinase inhibitor saracatinib is associated with the reactivation of the mammalian target of rapamycin pathway ${ }^{61,62}$. These results suggest that cancer cells can acquire resistance to Src inhibitor via multiple genetic alterations. Thus further clinical studies are needed to develop more reliable biomarkers that can guide clinical trials. To our knowledge, KRAS mutational status has not been evaluated as a biomarker for response to Src inhibitors. In light of our present study suggesting tumor-suppressive role of Src in the context of oncogenic KRAS as well as recent reports demonstrating clinical utility of SHP2 inhibitors in the management of mutant KRAS-driven cancers ${ }^{7-11}$, inclusion of KRAS mutational status would be prudent for future clinical trials of Src and SHP2 inhibitors as mono or combination therapies.

\section{Methods}

Cells. HEK293, MEF, MEF-SYF(-/-), CFPAC1, Capan-1, HPAF-II, SW1990, HUPT3, and MiaPaCa-2 cells were obtained from the American Type Culture Collection. P411T1 and PancT6 were generated from PDAC PDXs ${ }^{42}$. HEK293, MEF, MEF-SYF $(-/-)$, and MiaPaCa-2 cells were maintained in Dulbecco's modified Eagle's medium (DMEM; Invitrogen) supplemented with 10\% (v/v) heatinactivated fetal bovine serum (FBS; Wisent) at $37^{\circ} \mathrm{C}$ in a humidified $5 \% \mathrm{CO}_{2}$ atmosphere. CFPAC1, Capan-1, HPAF-II, SW1990, HPAC, and HUPT3 cells were maintained similarly in RPMI-1640 (Wisent) medium supplemented with $10 \%$ (v/ v) FBS. P411T1 and PancT6 cells were maintained in DMEM/F12 medium (Thermo Fisher Scientific, 11330-032) supplemented with $5 \mathrm{ng} / \mathrm{ml}$ EGF (R\&D Systems, 236-EG-01M), $10 \mu \mathrm{g} / \mathrm{ml}$ insulin (Thermo Fisher Scientific, 12585-014), and $10 \%(\mathrm{v} / \mathrm{v})$ FBS.

Plasmids. A plasmid encoding human pBabe-KRAS4B WT was generously provided by Channing Der (University of North Carolina, Chapel Hill), which was subcloned into pcDNA3 using KpnI and NotI to integrate an N-terminal HA tag. KRAS mutants (G12V, Y32F and Y64F) were generated by site-directed mutagenesis. Human pcDNA3-HA-HRAS, pCGN-HA-NRAS, pcDNA3-Flag-RAC2,
pCDNA-HA-FAK P712/715A, pCDNA-HA-FAK Y576/577F, pMSCV-mCherrySYK, pCMV5-Src (WT or K295RY527F), and pCMV5-SHP2 (WT, E76K or C459S) were obtained from Addgene. Flag-SHP2 constructs were subcloned into pcDNA3 and a plasmid encoding HA-CBL was subcloned into the pcDNADEST4.0 vector using Gateway Cloning technology (Invitrogen). Plasmids were verified by direct DNA sequencing.

Antibodies. Rabbit polyclonal antibodies against Src (\#2109, 1:5000), phosphorylated (p)Src (\#2101, 1:1000), pAKT (\#9271, 1:1000), AKT (\#9272, 1:1000), ERK (\#9102, 1:1000), SYK (\#2712, 1:1000), FAK (\#3285, 1:1000), pTyr (P-Tyr1000) (\#8954, 1:2000), cleaved caspase-3 (\#9664, 1:1000), cleaved PARP (\#9541, 1:1000), PARP (\#9542, 1:1000), cleaved caspase-9 (\#9661, 1:1000), caspase-9 (\#9508, 1:1000), and HA (\#3724, 1:5000) were obtained from Cell Signaling Technologies. Polyclonal IgG (sc-2027), pERK (sc-7383, 1:500), CBL (sc-170, 1:500), SHP2 (sc-280, 1:1000), pMEK1/2 (sc-81503, 1:500), and MEK-1 (sc-6250, 1:500) were obtained from Santa Cruz Biotechnology. p-SHP2(Y542) (ab62322, 1:20,000) was obtained from Abcam. Monoclonal antibodies against Pan-Ras (OP40, 1:500), HA (12CA5, 1:500), and pTyr (4G10) (05-321, 1:1000) were obtained from Boehringer Ingelheim and Millipore, respectively. Monoclonal FLAG-M2 (F1804, 1:2000) and Vinculin (V9264, 1:2000) were obtained from Sigma.

Chemicals. Compound 11a-1, 6-Hydroxy-3-iodo-1-methyl-2-(3-(2-oxo-2-((4(thiophen-3-yl)-phenyl)amino)acetamido)phenyl)-1H-indole-5-carboxylic acid was developed and synthesized using a structure-guided and fragment-based library approach ${ }^{14}$. SHP099 was obtained from Novartis. KX2-391 was obtained from Selleck Chemicals. Calf intestinal alkaline phosphatase was obtained from New England Biolabs. EGF and PDGF-BB were obtained from R\&D Systems.

CRISPR/Cas9-mediated gene editing. pLentiCRISPR (49535) was obtained from Addgene, and the following sequences derived from exon 1 of the indicated genes were used to create guides: SHP2 (mouse), 5'-CTGAACCAGTTCAGCCAAAG; SHP2 (human), 5'-GAGACTTCACACTTTCCGTT; and Non Target, 5'GCGAGGTATTCGGCTCCGCG. The cells were infected with lentivirus as described below.

Lentiviral production and infection of cell lines. HEK293FT cells (Thermo Fisher Scientific) were transfected with psPAX2, pMDG1.vsvg, and pLentiCRISPR transfer vector. Lentivirus containing supernatant was collected at $72 \mathrm{~h}$ posttransfection. Lentiviral supernatant was filtered and applied to the indicated cell lines. MEF and CFPAC1 cells required the addition of $5 \mu \mathrm{g} / \mathrm{ml}$ Polybrene (Millipore). Selection was started $24 \mathrm{~h}$ after infection using puromycin $(5 \mu \mathrm{g} / \mathrm{ml}$; Wisent). Monoclonal populations were generated and used for experiments.

Src knockdown via siRNA. Endogenous Src in SHP2-/- MEF cells was silenced using ON-TARGETplus SMARTpool siRNA (Dharmacon) according to the manufacturer's instructions.

Immunoprecipitation and immunoblotting. Cells were harvested in EBC lysis buffer $(50 \mathrm{mM}$ Tris, $\mathrm{pH} 8,120 \mathrm{mM} \mathrm{NaCl}, 0.5 \% \mathrm{NP}-40)$ supplemented with protease and phosphatase inhibitors (Roche). Lysates were immunoprecipitated using the indicated antibodies along with protein A-Sepharose (Repligen). Bound proteins were washed five times in NETN buffer $(20 \mathrm{mM}$ Tris, $\mathrm{pH} 8,100 \mathrm{mM} \mathrm{NaCl}, 1$ mM EDTA, $0.5 \%$ NP-40), eluted by boiling in sample buffer, and resolved by SDSPAGE. Proteins were electrotransferred onto polyvinylidene difluoride membrane (Bio-Rad), blocked, and probed with the indicated antibodies.

Cellular RAS activity assay. RAS activity was assessed using the RAS activation assay kit from Millipore (17-218). Briefly, RAS-GTP from various treated lysates was pulled-down using an agarose-bound glutathione $S$-transferase (GST) fusion protein corresponding to human RBD of RAF-1. The presence of RAS-GTP was detected by western blotting using an anti-RAS antibody (Millipore \# 05-516, 1:2000). 
Cell proliferation assay. Equal numbers of cells were plated in quadruplicate in 96-well plates in the presence or absence of the indicated inhibitors and cellular proliferation was assessed using Alamar Blue proliferation assay as per the manufacturer's instructions (Invitrogen).

Recombinant protein expression. The kinase domain from Src was produced recombinantly in Escherichia coli ${ }^{63}$. Briefly, DNA corresponding to Src (residues 254-536) was subcloned into pET46 Ek/LIC. Additionally, DNA corresponding to full-length Yersinia pestis YopH phosphatase was subcloned into pRSF Ek/LIC. In both constructs, a thrombin protease site was introduced after the vector encoded $\mathrm{N}$-terminal 6-histidine purification tag. Soluble expression of Src was achieved by co-expression of YopH in Rosetta-2 (DE3) E. coli cells. Cell cultures were grown to an $\mathrm{OD}_{600}=0.8$ at $37^{\circ} \mathrm{C}$ and recombinant protein expression was induced with a final concentration of $0.5 \mathrm{mM} \mathrm{IPTG}$ for $20 \mathrm{~h}$ at $18^{\circ} \mathrm{C}$. Pelleted cells were resuspended in $50 \mathrm{mM}$ Tris- $\mathrm{HCl} \mathrm{pH} \mathrm{8.0,500} \mathrm{mM} \mathrm{NaCl,} 25 \mathrm{mM}$ imidazole, and $5 \%(\mathrm{v} / \mathrm{v})$ glycerol. E. coli cells were lysed using a hydraulic cell disruption system (Constant Systems) and recombinant protein was purified by standard Ni-NTA affinity chromatography. Pooled elutions containing both Src and YopH were then dialyzed overnight against 40 volumes of buffer containing $20 \mathrm{mM}$ Tris- $\mathrm{HCl} \mathrm{pH} \mathrm{8.0,}$ $100 \mathrm{mM} \mathrm{NaCl}, 5 \%(\mathrm{v} / \mathrm{v})$ glycerol, and $1 \mathrm{mM}$ dithiothreitol (DTT). Following overnight dialysis, the protein solution was applied onto an anion exchange column (Mono Q 5/50 GL) equilibrated with $20 \mathrm{mM}$ Tris-HCl pH 8.0, 5\% (v/v) glycerol, and $1 \mathrm{mM}$ DTT (Buffer A). Protein bound to the anion exchange column was eluted with a $100 \mathrm{CV}$ gradient of $0-50 \% 20 \mathrm{mM}$ Tris- $\mathrm{HCl} \mathrm{pH} 8.0,1 \mathrm{M} \mathrm{NaCl}$, $5 \%(\mathrm{v} / \mathrm{v})$ glycerol, and $1 \mathrm{mM}$ DTT (Buffer B). Anion exchange was sufficient for the separation of recombinant Src kinase from the YopH phosphatase. The elutions containing Src kinase were pooled and further purified by size exclusion chromatography using a custom Superdex-200 10/300 prep grade column equilibrated in $50 \mathrm{mM}$ Tris- $\mathrm{HCl}, \mathrm{pH} 8.0,100 \mathrm{mM} \mathrm{NaCl}, 5 \%$ (v/v) glycerol, and $1 \mathrm{mM}$ DTT. All purification steps were carried out at $4{ }^{\circ} \mathrm{C}$. Protein concentration was determined by absorbance at $\lambda=280 \mathrm{~nm}$, and purity was confirmed by SDS-PAGE and MS.

The catalytic regions of Son of Sevenless SOScat (residues 564-1049) and GAP domain of human RASA1 (residues 715-1074) were subcloned into pET15b (Novagen/EMD Biosciences). RBD of BRAF (residues 150-233) fused with Nterminal GST tag was subcloned into pGEX-4T2 (HE Healthcare). The proteins were expressed in E. coli BL21 (DE3). GST-RBD was purified using Glutathione Sepharose 4B (GE Healthcare) followed by gel filtration chromatography (Superdex ${ }^{\text {tw }}$ S200, GE Healthcare). His-tagged SOS ${ }^{\text {cat }}$ and RASA1 GAP domain were purified using Ni-NTA (Qiagen). The His tag was cleaved by thrombin following elution with $250 \mathrm{mM}$ imidazole, and final purification was achieved by gel filtration chromatography (Superdex ${ }^{\text {mit }}$ S75, GE Healthcare) ${ }^{64}$.

Purification of isotopically labeled $\left({ }^{15} \mathbf{N}\right)$ KRAS. A synthetic gene (Genscript) encoding the GTPase domain of KRAS4B (residues 1-173, with C118S mutation) was cloned with a thrombin-cleavable His-tag into pET-28 and transformed into $E$. coli BL21 (DE3). The bacteria were cultured in minimal M9 medium supplemented with $1 \mathrm{~g} / 1{ }^{15} \mathrm{~N}$ ammonium chloride at $37^{\circ} \mathrm{C}$ until the O.D. 600 reached 0.6 , then induced with $0.2 \mathrm{mM}$ IPTG (isopropyl $\beta$-D-1-thiogalactopyranoside) at $16^{\circ} \mathrm{C}$ overnight. The cells were harvested, resuspended in lysis buffer (50 mM Tris, 150 $\mathrm{mM} \mathrm{NaCl}, 0.1 \% \mathrm{NP}-40,10 \%$ Glycerol, $10 \mathrm{mM}$ Imidazole, $5 \mathrm{mM} \mathrm{MgCl}, 1 \mathrm{mM}$ phenylmethylsulfonyl fluoride, $10 \mathrm{mM} \beta$-mercaptoethanol and lysozyme at $\mathrm{pH}$ 8.0), and lysed by sonication. The protein was purified using Ni-NTA resin followed by gel filtration (Superdex ${ }^{\text {six }}$ S75, GE Healthcare) in a buffer containing 20 $\mathrm{mM}$ HEPES, $100 \mathrm{mM} \mathrm{NaCl}, 5 \mathrm{mM} \mathrm{MgCl}$, and $2 \mathrm{mM}$ tris(2-carboxyethyl)phosphine (TCEP), pH 7.4. KRAS copurifies with $E$. coli-derived guanosine nucleotide, and upon completion of purification, the WT protein is loaded with GDP. KRAS $\mathrm{G} 12 \mathrm{~V}$ was purified in the same manner; however, it comprises a mixture of GDPand GTP-bound protein due to its impaired GTP hydrolysis, thus it was incubated for several days to allow GTP hydrolysis to proceed to completion.

In vitro kinase assay. Purified recombinant human KRAS WT was incubated with purified recombinant active His-Src in $100 \mu \mathrm{l}$ of kinase buffer [50 mM HEPES (pH7.5), $10 \mathrm{mM} \mathrm{MgCl}_{2}, 1 \mathrm{mM}$ EGTA, $0.01 \%$ Brij-35, $200 \mu \mathrm{M}$ ATP] for $1 \mathrm{~h}$ at room temperature. Thereafter, $1 \mathrm{ml}$ of binding buffer [50 mM Tris (pH 8), $120 \mathrm{mM} \mathrm{NaCl}$, $0.1 \%$ Nonidet P-40, $5 \%(\mathrm{v} / \mathrm{v})$ glycerol] was added along with the indicated antibodies and protein A-Sepharose (Repligen). Bound proteins were washed five times in binding buffer before elution by boiling in sample buffer and resolved by SDSPAGE.

Real-time NMR GTPase assay. To prepare tyrosyl-phosphorylated KRAS for GTPase assays, a sample of ${ }^{15} \mathrm{~N}$-KRAS was confirmed to be GDP-loaded by collecting a ${ }^{1} \mathrm{H}-{ }^{15} \mathrm{~N}$ HSQC spectrum, then incubated with a catalytic amount of recombinant $\mathrm{Src}$ (i.e., 1:125 molar ratio) in the presence of $2 \mathrm{mM}$ ATP, $1 \mathrm{mM}$ activated sodium vanadate, $2 \mathrm{mM}$ imidazole, $1 \mathrm{mM}$ sodium fluoride, and $1.15 \mathrm{mM}$ sodium molybdate. After confirming completion of the phosphorylation reaction by collection of a ${ }^{1} \mathrm{H}^{15} \mathrm{~N}$ HSQC spectrum, the Src protein and excess ATP was removed from the KRAS sample by gel filtration chromatography (Superdex ${ }^{\mathrm{mm}}$ S75 GE Healthcare). For nucleotide exchange assays, $40 \mu \mathrm{l}$ of $250 \mu \mathrm{M}$ GDP-loaded ${ }^{15} \mathrm{~N}$ KRAS or phosphorylated ${ }^{15} \mathrm{~N}$-KRAS was incubated with $10 \times$ molar excess GTP $\gamma \mathrm{S}$ (guanosine $5^{\prime}$-[ $\gamma$-thio]triphosphate tetralithium salt, Sigma-Aldrich) in a $1.7 \mathrm{~mm}$ NMR tube. Sequential ${ }^{1} \mathrm{H}^{-15} \mathrm{~N}$ HSQC NMR experiments were collected throughout the time course of the exchange reaction using a Bruker $600 \mathrm{MHz}$ Avance III NMR spectrometer equipped with a $1.7 \mathrm{~mm}$ cryogenic TCI MicroCryoProbe. Data were processed using NMRPipe and analyzed using the NMRFAM-SPARKY software ${ }^{65,66}$. To calculate the nucleotide exchange rate, the fraction of RAS bound to GDP based on the ratio of the peak intensities of three cross-peaks sensitive to nucleotide exchange were plotted vs. time and fit to an exponential association curve ${ }^{67}$ using GraphPad Prism 4. GEF assays were performed in the same manner with the addition of catalytic domain of Son of Sevenless (residues 564-1049 SOScat) at a molar ratio of 1:600 to KRAS. To perform KRAS GTP hydrolysis assays, ${ }^{15} \mathrm{~N}$-KRAS was loaded with GTP by incubation with a 10 -fold excess of GTP in the presence of EDTA, which were removed by gel filtration chromatography (Superdex 75 10/300, GE Healthcare), before collecting sequential HSQC spectra. To perform GAP assays, recombinant GAP domain of RASA1 (a construct known as GAP-334) was added at a 1:3000 molar ratio to $40 \mu \mathrm{l}$ of $250 \mu \mathrm{M}^{15} \mathrm{~N}$ KRAS loaded with GTP. The sample was then placed in a $1.7 \mathrm{~mm}$ NMR tube to acquire sequential ${ }^{1} \mathrm{H}-{ }^{15} \mathrm{~N}$ HSQC spectra during the GTP hydrolysis reaction. The NMR data were processed and analyzed in a manner similar to that described above for nucleotide exchange.

Mass analysis of intact pKRAS. KRAS and pKRAS samples in $20 \mathrm{mM}$ HEPES, $100 \mathrm{mM} \mathrm{NaCl}, 5 \mathrm{mM} \mathrm{MgCl}$, $2 \mathrm{mM}$ TCEP, $1 \mathrm{mM}$ activated sodium vanadate, 2 $\mathrm{mM}$ imidazole, $1 \mathrm{mM}$ sodium fluoride, and $1.15 \mathrm{mM}$ sodium molybdate at $\mathrm{pH} 7.4$ were diluted 1 to 5 with $20 \mathrm{mM}$ Tris-Base, $5 \mathrm{mM} \mathrm{MgCl} 2,2 \mathrm{mM}$ TCEP, pH 5.5 to obtain a final concentration of $50 \mu \mathrm{M}$. The accurate mass of these samples were obtained using an Agilent 6538 Ultra High Definition (UHD) Quadrupole time-offlight mass spectrometer run in positive mode with electrospray ionization.

Ion exchange chromatography. The mono-, di-, and un-phosphorylated forms of KRAS were separated by anion exchange chromatography using a Mono Q 5/50 GL column run with $20 \mathrm{mM}$ HEPES pH 7.0, $5 \mathrm{mM} \mathrm{MgCl}_{2}$, and $1 \mathrm{mM}$ TCEP (Buffer A) and $20 \mathrm{mM}$ HEPES pH 7.0, $5 \mathrm{mM} \mathrm{MgCl}_{2}, 1 \mathrm{mM}$ TCEP, and $1 \mathrm{M} \mathrm{NaCl}$ (Buffer B). The separation was achieved with an 80 column volume gradient of $0-40 \%$ buffer B. Both the wash buffer and elution buffer contained phosphatase inhibitors ( $1 \mathrm{mM}$ activated sodium vanadate, $2 \mathrm{mM}$ imidazole, $1 \mathrm{mM}$ sodium fluoride, and $1.15 \mathrm{mM}$ sodium molybdate) to stabilize the phosphorylated forms of KRAS.

Liquid chromatography and MS. Kinase assay samples were reduced with DTT (5 $\mathrm{mM})$ and alkylated with iodoacetamide $(10 \mathrm{mM})$ followed by overnight digestion with trypsin $\left(10 \mu \mathrm{g} / \mathrm{ml}\right.$ in $\left.50 \mathrm{mM} \mathrm{NH}{ }_{4} \mathrm{HCO}_{3}\right)$ at $37^{\circ} \mathrm{C}$. The resulting peptides were de-salted using reversed-phase $\mathrm{C} 18$ columns and lyophilized in a vacuum centrifuge. Samples were reconstituted in $0.1 \% \mathrm{HCOOH}$ and analyzed by LC-MS. Using an EASY-nLC 1000 pump, samples were loaded in-line on an Acclaim PepMap $^{\mathrm{TM}} 100$ nanoViper pre-column $(75 \mu \mathrm{m} \times 2 \mathrm{~cm}, 3 \mu \mathrm{m})$ and resolved on an Acclaim PepMap ${ }^{\text {TM }}$ RSLC nanoViper analytical column $(75 \mu \mathrm{m} \times 50 \mathrm{~cm}, 3 \mu \mathrm{m})$ over a 120 -min acetonitrile gradient $(0-40 \%)$ in a $0.1 \% \mathrm{HCOOH}$ mobile phase. Positivemode electrospray ionization was applied and ions were analyzed by MS using a QExactive HF instrument set to perform MS/MS HCD fragmentation scans on up to the 20 most intense ions (minimum ion count of 1000 for activation) from an MS parent ion scan $(390-1800 \mathrm{~m} / \mathrm{z}$ range; 60,000 full-width half-maximum resolution @ $200 \mathrm{~m} / \mathrm{z}$ ). Fragmented ions were placed on a dynamic exclusion list for $5 \mathrm{~s}$. Acquired raw files were converted to the.mgf format using Proteowizard (v3.0.10800), then searched using X!Tandem (v2013.06.15.1) against Human RefSeq Version 45 (36,113 entries). Search parameters specified a parent ion tolerance of $15 \mathrm{ppm}$ and a fragment ion tolerance of $0.4 \mathrm{Da}$, with one missed cleavage allowed for trypsin. Carbamidomethylation [C] was set as a fixed modification and oxidation $[\mathrm{M}]$, deamidation $(\mathrm{N}, \mathrm{Q})$, acetylation (Protein N-term), and phosphorylation (STY) were allowed as variable modifications. All data are publicly available and have been uploaded to the MassIVE archive (https://massive.ucsd.edu).

Biolayer interferometry. The affinity of KRAS and pKRAS was determined using an Octet RED-384 biolayer interferometry instrument equipped with the Octet Data Acquisition 9.0.0.37 and FortéBio Data analysis software (Pall). The assay was performed using 96-well plates at $25^{\circ} \mathrm{C}$ with $1000 \mathrm{rpm}$ agitation in a buffer comprising $20 \mathrm{mM}$ HEPES, $100 \mathrm{mM} \mathrm{NaCl}, 5 \mathrm{mM} \mathrm{MgCl}$, and $2 \mathrm{mM}$ TCEP supplemented with $1 \%$ BSA and $0.005 \%$ Tween-20 to minimize nonspecific binding and cocktail of phosphatase inhibitors $(1 \mathrm{mM}$ activated sodium vanadate, $2 \mathrm{mM}$ imidazole, $1 \mathrm{mM}$ sodium fluoride, and $1.15 \mathrm{mM}$ sodium molybdate). GST-tagged BRAF-RBD (residues $150-233)(2.5 \mu \mathrm{g} / \mathrm{ml})$ was immobilized to anti-GSTconjugated biosensors (Pall FortéBio), which were then dipped into wells containing increasing concentrations of KRAS for $30 \mathrm{~s}$, followed by a dissociation step in buffer. A sensor with immobilized BRAF-RBD was dipped into buffer only to subtract instrument drift. The data were analyzed and fitted for global analysis to obtain $K_{\mathrm{D}}$ values assuming one-to-one stoichiometry.

3D spheroid assay. In vitro 3D spheroid culture was performed using an UltraLow Attachment surface coating 96-well spheroid microplate (Corning). Briefly, 
equal numbers of cells were plated in 96-well spheroid microplates and cultured in the presence or absence of the indicated inhibitors. Spheroid viability was determined using the CellTiter-Glo 3D Cell viability assay according to the protocol provided by the manufacturer.

Organoid cultures. PDAC organoid models used in this study were generated by the Princess Margaret Living Biobank (PMLB) Organoid core facility (https://www. livingbiobank.ca/) from PDX tissues as per the published protocols ${ }^{68}$. All organoids used in this study were characterized extensively by PMLB using the following guidelines. Short tandem repeat (STR) profiling was performed on DNA isolated from the organoid culture and the profile was compared with the STR profile of the primary patient material. Second, organoid culture was embedded and histopathological analysis was performed to assess the morphological features. Immunohistochemistry was performed using antibodies that are clinically relevant to the organ site. The doubling rate of each organoid was determined using short-term viability assays and Cell Titer Glo. Flow analysis using EPCAM antibodies was also performed to ensure the epithelial origin of the organoids. Finally, mycoplasma test was performed on all the established organoid models.

Organoid drug screening. Organoids were dissociated to single cells and seeded on top of a thin layer of Matrigel in 384-well plate (3000/well). Next day, 11a-1 or SHP099 was added in a six-point concentration series to wells in triplicate. Cell viability was assessed by ATP quantification using the CellTiter-Glo 3D luminescence-based assay. Viability values were normalized to vehicle control wells. Experiment was repeated three times.

Tumor xenograft experiments. The University Health Network (UHN) Animal Care Committee approved the animal study protocols. Thirteen male SCID mice aged 4-6 weeks were implanted with treatment-naive tumors generated from pancreatic-derived xenograft model OCIP. 343 obtained from the PMLB Core Facility (Toronto, Canada). When tumor size reached $200 \mathrm{~mm}^{3}$, the mice were randomized into three treatment groups; control $(n=5)$, SHP099 $(n=5)$, and Trametinib $(n=3)$. SHP099 $(100 \mathrm{mg} / \mathrm{kg})$ and Trametinib $(1 \mathrm{mg} / \mathrm{kg})$ were administered via oral gavage daily. Tumor growth was measured twice per week until they reached $1.5 \mathrm{~cm}$ in diameter. Tumor volume was calculated as follows: length $\times$ width $^{2} \times 0.52$. At the end of the experiment, mice were sacrificed and tumor tissues were harvested for either formalin fixation or immediate snap freezing in liquid nitrogen.

Statistical analyses. Unpaired two-tailed Student's $t$ test was used to compare between treatment groups and cell types. All statistical analysis was performed using the GraphPad PRISM 6.0 software unless otherwise stated. $P$ value $<0.05$ was considered statistically significant.

Reporting summary. Further information on experimental design is available in the Nature Research Reporting Summary linked to this article.

\section{Data availability}

Data supporting the findings of this manuscript are available from the corresponding author upon reasonable request. The MS data are publicly available and have been uploaded to the MassIVE archive (https://massive.ucsd.edu). The Source Data underlying Figs. 1f, 3c, d, 5b-e, and $6 \mathrm{~b}$ and Supplementary Figs. 1a, b, e, f, h, $2 \mathrm{~b}, \mathrm{c}, 3 \mathrm{a}, \mathrm{b}$, and $7 \mathrm{a}, \mathrm{b}$ are provided as a Source Data file.

Received: 27 August 2018 Accepted: 17 December 2018

Published online: 15 January 2019

\section{References}

1. Barbacid, M. ras genes. Annu. Rev. Biochem. 56, 779-827 (1987).

2. Kano, Y., Cook, J. D., Lee, J. E. \& Ohh, M. New structural and functional insight into the regulation of Ras. Semin. Cell Dev. Biol. 58, 70-78 (2016).

3. Campbell, S. L., Khosravi-Far, R., Rossman, K. L., Clark, G. J. \& Der, C. J. Increasing complexity of Ras signaling. Oncogene 17, 1395-1413 (1998).

4. Hunter, J. C. et al. Biochemical and structural analysis of common cancerassociated KRAS mutations. Mol. Cancer Res. 13, 1325-1335 (2015).

5. Bunda, S. et al. Src promotes GTPase activity of Ras via tyrosine 32 phosphorylation. Proc. Natl Acad. Sci. USA 111, E3785-E3794 (2014).

6. Bunda, S. et al. Inhibition of SHP2-mediated dephosphorylation of Ras suppresses oncogenesis. Nat. Commun. 6, 8859 (2015).

7. Mainardi, S. et al. SHP2 is required for growth of KRAS-mutant non-smallcell lung cancer in vivo. Nat. Med. 24, 961-967 (2018).

8. Ruess, D. A. et al. Mutant KRAS-driven cancers depend on PTPN11/SHP2 phosphatase. Nat. Med. 24, 954-960 (2018).
9. Fedele, C. et al. SHP2 inhibition prevents adaptive resistance to MEK inhibitors in multiple cancer models. Cancer Discov. 8, 1237-1249 (2018).

10. Wong, G. S. et al. Targeting wild-type KRAS-amplified gastroesophageal cancer through combined MEK and SHP2 inhibition. Nat. Med. 24, 968-977 (2018).

11. Nichols, R. J. et al. RAS nucleotide cycling underlies the SHP2 phosphatase dependence of mutant BRAF-, NF1- and RAS-driven cancers. Nat. Cell Biol. 20, 1064-1073 (2018).

12. Grewal, T., Koese, M., Tebar, F. \& Enrich, C. Differential regulation of RasGAPs in cancer. Genes Cancer 2, 288-297 (2011).

13. Cox, A. D., Fesik, S. W., Kimmelman, A. C., Luo, J. \& Der, C. J. Drugging the undruggable RAS: mission possible? Nat. Rev. Drug Discov. 13, 828-851 (2014).

14. Zeng, L. F. et al. Therapeutic potential of targeting the oncogenic SHP2 phosphatase. J. Med. Chem. 57, 6594-6609 (2014).

15. Chen, Y. N. et al. Allosteric inhibition of SHP2 phosphatase inhibits cancers driven by receptor tyrosine kinases. Nature 535, 148-152 (2016).

16. Theillet, F. X. et al. Cell signaling, post-translational protein modifications and NMR spectroscopy. J. Biomol. NMR 54, 217-236 (2012).

17. Bunda, S. et al. Inhibition of SRC corrects GM-CSF hypersensitivity that underlies juvenile myelomonocytic leukemia. Cancer Res. 73, 2540-2550 (2013).

18. Kinoshita, E., Kinoshita-Kikuta, E. \& Koike, T. Separation and detection of large phosphoproteins using Phos-tag SDS-PAGE. Nat. Protoc. 4, 1513-1521 (2009).

19. Naing, A. et al. A phase I trial of KX2-391, a novel non-ATP competitive substrate-pocket- directed SRC inhibitor, in patients with advanced malignancies. Invest. New Drugs 31, 967-973 (2013).

20. Marshall, C. B. et al. Characterization of the intrinsic and TSC2-GAPregulated GTPase activity of Rheb by real-time NMR. Sci. Signal. 2, ra3 (2009).

21. Smith, M. J., Neel, B. G. \& Ikura, M. NMR-based functional profiling of RASopathies and oncogenic RAS mutations. Proc. Natl Acad. Sci. USA 110, 4574-4579 (2013)

22. Vigil, D., Cherfils, J., Rossman, K. L. \& Der, C. J. Ras superfamily GEFs and GAPs: validated and tractable targets for cancer therapy? Nat. Rev. Cancer 10, 842-857 (2010).

23. Zhang, R. Y. et al. SHP2 phosphatase as a novel therapeutic target for melanoma treatment. Oncotarget 7, 73817-73829 (2016)

24. Zheng, J. et al. Expression and prognosis value of SHP2 in patients with pancreatic ductal adenocarcinoma. Tumour Biol. 37, 7853-7859 (2016).

25. Zheng, J. et al. Pancreatic cancer risk variant in LINC00673 creates a miR1231 binding site and interferes with PTPN11 degradation. Nat. Genet. 48, 747-757 (2016).

26. Mohi, M. G. \& Neel, B. G. The role of Shp2 (PTPN11) in cancer. Curr. Opin. Genet. Dev. 17, 23-30 (2007)

27. Bennett, A. M., Tang, T. L., Sugimoto, S., Walsh, C. T. \& Neel, B. G. Protein tyrosine-phosphatase SHPTP2 couples platelet-derived growth factor receptor beta to Ras. Proc. Natl Acad. Sci. USA 91, 7335-7339 (1994).

28. Li, W. et al. A new function for a phosphotyrosine phosphatase: linking GRB2-Sos to a receptor tyrosine kinase. Mol. Cell. Biol. 14, 509-517 (1994).

29. Dance, M., Montagner, A., Salles, J. P., Yart, A. \& Raynal, P. The molecular functions of Shp2 in the Ras/Mitogen-activated protein kinase (ERK1/2) pathway. Cell. Signal. 20, 453-459 (2008).

30. Agazie, Y. M. \& Hayman, M. J. Molecular mechanism for a role of SHP2 in epidermal growth factor receptor signaling. Mol. Cell. Biol. 23, 7875-7886 (2003).

31. Montagner, A. et al. A novel role for Gab1 and SHP2 in epidermal growth factor-induced Ras activation. J. Biol. Chem. 280, 5350-5360 (2005).

32. Hanafusa, H., Torii, S., Yasunaga, T., Matsumoto, K. \& Nishida, E. Shp2, an $\mathrm{SH} 2$-containing protein-tyrosine phosphatase, positively regulates receptor tyrosine kinase signaling by dephosphorylating and inactivating the inhibitor Sprouty. J. Biol. Chem. 279, 22992-22995 (2004).

33. Uezu, A. et al. Modified SH2 domain to phototrap and identify phosphotyrosine proteins from subcellular sites within cells. Proc. Natl Acad. Sci. USA 109, E2929-E2938 (2012).

34. Ding, J., Soule, G., Overmeyer, J. H. \& Maltese, W. A. Tyrosine phosphorylation of the Rab24 GTPase in cultured mammalian cells. Biochem. Biophys. Res. Commun. 312, 670-675 (2003).

35. Brucker, S., Gerwert, K. \& Kotting, C. Tyr39 of ran preserves the Ran.GTP gradient by inhibiting GTP hydrolysis. J. Mol. Biol. 401, 1-6 (2010).

36. Tu, S., Wu, W. J., Wang, J. \& Cerione, R. A. Epidermal growth factordependent regulation of $\mathrm{Cdc} 42$ is mediated by the Src tyrosine kinase. J. Biol. Chem. 278, 49293-49300 (2003).

37. Zou, J. X., Liu, Y., Pasquale, E. B. \& Ruoslahti, E. Activated SRC oncogene phosphorylates R-ras and suppresses integrin activity. J. Biol. Chem. 277, 1824-1827 (2002). 
38. Hausdorff, W. P. et al. Tyrosine phosphorylation of $\mathrm{G}$ protein alpha subunits by pp60c-src. Proc. Natl Acad. Sci. USA 89, 5720-5724 (1992).

39. Ting, P. Y. et al. Tyrosine phosphorylation of RAS by ABL allosterically enhances effector binding. FASEB J. 29, 3750-3761 (2015).

40. Sveshnikova, N., Soll, J. \& Schleiff, E. Toc34 is a preprotein receptor regulated by GTP and phosphorylation. Proc. Natl Acad. Sci. USA 97, 4973-4978 (2000).

41. Jelic, M. et al. The chloroplast import receptor Toc34 functions as preproteinregulated GTPase. Biol. Chem. 383, 1875-1883 (2002).

42. Moffitt, R. A. et al. Virtual microdissection identifies distinct tumor- and stroma-specific subtypes of pancreatic ductal adenocarcinoma. Nat. Genet. 47, 1168-1178 (2015).

43. Boriack-Sjodin, P. A., Margarit, S. M., Bar-Sagi, D. \& Kuriyan, J. The structural basis of the activation of Ras by Sos. Nature 394, 337-343 (1998).

44. Sondermann, H. et al. Structural analysis of autoinhibition in the Ras activator Son of sevenless. Cell 119, 393-405 (2004).

45. Hall, B. E., Yang, S. S., Boriack-Sjodin, P. A., Kuriyan, J. \& Bar-Sagi, D. Structure-based mutagenesis reveals distinct functions for Ras switch 1 and switch 2 in Sos-catalyzed guanine nucleotide exchange. J. Biol. Chem. 276, 27629-27637 (2001).

46. Frech, M. et al. Role of glutamine-61 in the hydrolysis of GTP by p21H-ras: an experimental and theoretical study. Biochemistry 33, 3237-3244 (1994).

47. Pai, E. F. et al. Refined crystal structure of the triphosphate conformation of H-ras p21 at 1.35 A resolution: implications for the mechanism of GTP hydrolysis. EMBO J. 9, 2351-2359 (1990).

48. Fetics, S. K. et al. Allosteric effects of the oncogenic RasQ61L mutant on RafRBD. Structure 23, 505-516 (2015)

49. Ford, B., Hornak, V., Kleinman, H. \& Nassar, N. Structure of a transient intermediate for GTP hydrolysis by ras. Structure 14, 427-436 (2006).

50. Hall, B. E., Bar-Sagi, D. \& Nassar, N. The structural basis for the transition from Ras-GTP to Ras-GDP. Proc. Natl Acad. Sci. USA 99, 12138-12142 (2002).

51. Scheffzek, K. et al. The Ras-RasGAP complex: structural basis for GTPase activation and its loss in oncogenic Ras mutants. Science 277, 333-338 (1997).

52. Nur, E. K. M. S., Sizeland, A., D’Abaco, G. \& Maruta, H. Asparagine 26, glutamic acid 31, valine 45, and tyrosine 64 of Ras proteins are required for their oncogenicity. J. Biol. Chem. 267, 1415-1418 (1992).

53. Yamasaki, K. et al. Site-directed mutagenesis, fluorescence, and twodimensional NMR studies on microenvironments of effector region aromatic residues of human c-Ha-Ras protein. Biochemistry 33, 65-73 (1994).

54. Buhrman, G., Holzapfel, G., Fetics, S. \& Mattos, C. Allosteric modulation of Ras positions Q61 for a direct role in catalysis. Proc. Natl Acad. Sci. USA 107, 4931-4936 (2010).

55. Zhang, S. \& Yu, D. Targeting Src family kinases in anti-cancer therapies: turning promise into triumph. Trends Pharmacol. Sci. 33, 122-128 (2012).

56. Nagaraj, N. S., Smith, J. J., Revetta, F., Washington, M. K. \& Merchant, N. B. Targeted inhibition of SRC kinase signaling attenuates pancreatic tumorigenesis. Mol. Cancer Ther. 9, 2322-2332 (2010).

57. Arcaroli, J. J. et al. Gene array and fluorescence in situ hybridization biomarkers of activity of saracatinib (AZD0530), a Src inhibitor, in a preclinical model of colorectal cancer. Clin. Cancer Res. 16, 4165-4177 (2010).

58. Suwaki, N. et al. A HIF-regulated VHL-PTP1B-Src signaling axis identifies a therapeutic target in renal cell carcinoma. Sci. Transl Med. 3, 85ra47 (2011).

59. Okamoto, W. et al. Identification of c-Src as a potential therapeutic target for gastric cancer and of MET activation as a cause of resistance to c-Src inhibition. Mol. Cancer Ther. 9, 1188-1197 (2010).

60. Ahn, J. H. \& Lee, M. Suppression of autophagy sensitizes multidrug resistant cells towards Src tyrosine kinase specific inhibitor PP2. Cancer Lett. 310 188-197 (2011).

61. Chen, Y. et al. Combined Src and ER blockade impairs human breast cancer proliferation in vitro and in vivo. Breast Cancer Res. Treat. 128, 69-78 (2011).

62. Chen, Y. et al. Combined Src and aromatase inhibition impairs human breast cancer growth in vivo and bypass pathways are activated in AZD0530resistant tumors. Clin. Cancer Res. 15, 3396-3405 (2009).

63. Seeliger, M. A. et al. High yield bacterial expression of active c-Abl and c-Src tyrosine kinases. Protein Sci. 14, 3135-3139 (2005).

64. Smith, M. J. \& Ikura, M. Integrated RAS signaling defined by parallel NMR detection of effectors and regulators. Nat. Chem. Biol. 10, 223-230 (2014).

65. Delaglio, F. et al. NMRPipe: a multidimensional spectral processing system based on UNIX pipes. J. Biomol. NMR 6, 277-293 (1995).

66. Lee, W., Tonelli, M. \& Markley, J. L. NMRFAM-SPARKY: enhanced software for biomolecular NMR spectroscopy. Bioinformatics 31, 1325-1327 (2015).
67. Marshall, C. B. et al. Probing the GTPase cycle with real-time NMR: GAP and GEF activities in cell extracts. Methods 57, 473-485 (2012).

68. Boj, S. F. et al. Organoid models of human and mouse ductal pancreatic cancer. Cell 160, 324-338 (2015).

\section{Acknowledgements}

We thank the members of the Ohh and Ikura laboratories for helpful discussions and critical reading of this manuscript. We thank Jeremy Mogridge and Justin Maede (University of Toronto) for technical assistant with PhosTag SDS-PAGE analysis. We thank Wenguang He for the purification of recombinant Src and SHP2. We thank G. Gasmi-Seabrook for NMR expertise. This work was supported by funds from the Canadian Institutes of Health Research (FDN-1542284 to M.I. and MOP-119356 to M. O.), Canadian Cancer Society Research Institute (703209 to M.I.), Princess Margaret Foundation (to M.I.), NIH RO1 CA207288 (to Z.-Y.Z.), Program for Advancing Strategic International Networks to Accelerate the Circulation of Talented Researchers from Japan Society for the Promotion of Science and A Long-Term Overseas Research Fellowship from Sumitomo Life Welfare and Culture Foundation (to Y.K.) and Cancer Research Society, George and Helen Vari Foundation (to T.G.). The NMR facility at UHN is supported by Canada Foundation for Innovation.

\section{Author contributions}

Y.K., T.G., and C.B.M. designed, performed, and interpreted the experiments; conceptualized the project; and wrote the manuscript. N.R. designed, performed, and interpreted the organoid and tumor xenograft study. B.P.K.P. generated and performed experiments using plasmids encoding KRAS and SHP2 mutants. J.S.-G. designed, performed, and interpreted the MS study. J.D.C. designed and purified recombinant Src. I. V.-S. performed and interpreted cell viability experiments. B.M.M.G assisted NMR study. B.R. designed and interpreted the MS study. M.S.I. designed and interpreted cell viability experiments. J.E.L. designed and interpreted experiments involving recombinant Src. S.G. H. and J.J.Y. designed and interpreted PDX cell viability experiments. J.M. and Z.-Y.Z. designed and manufactured 11a-1 and interpreted SHP2 inhibitor study. M.S.T. designed and interpreted the organoid and tumor xenograft study. M.I. designed, interpreted, and wrote the NMR study. M.O. conceptualized the project, interpreted the data, and wrote the manuscript.

\section{Additional information}

Supplementary Information accompanies this paper at https://doi.org/10.1038/s41467 018-08115-8.

Competing interests: The authors declare no competing interests.

Reprints and permission information is available online at http://npg.nature.com/ reprintsandpermissions/

Journal peer review information: Nature Communications thanks John Colicelli, Hana Algül, and the other anonymous reviewer(s) for their contribution to the peer review of this work. Peer reviewer reports are available.

Publisher's note: Springer Nature remains neutral with regard to jurisdictional claims in published maps and institutional affiliations.

Open Access This article is licensed under a Creative Commons Attribution 4.0 International License, which permits use, sharing, adaptation, distribution and reproduction in any medium or format, as long as you give appropriate credit to the original author(s) and the source, provide a link to the Creative Commons license, and indicate if changes were made. The images or other third party material in this article are included in the article's Creative Commons license, unless indicated otherwise in a credit line to the material. If material is not included in the article's Creative Commons license and your intended use is not permitted by statutory regulation or exceeds the permitted use, you will need to obtain permission directly from the copyright holder. To view a copy of this license, visit http://creativecommons.org/ licenses/by/4.0/.

(C) The Author(s) 2019 